\title{
A comparative analysis due to the effect of point source on generation of $\mathrm{SH}$ wave
}

\author{
Shishir Gupta ${ }^{1}$, Snehamoy Pramanik ${ }^{1} *$ ๑, Smita $^{1}$ and Arun Kumar Verma ${ }^{2}$ \\ ${ }^{1}$ Department of Applied Mathematics, Indian Institute of Technology (Indian School of Mines), \\ Dhanbad 826004, India. \\ ${ }^{2}$ Department of Mathematics, Hampton University, Hampton, USA. \\ *Corresponding author.e-mail: snehamoy.pramanik@gmail.com
}

MS received 5 April 2018; revised 28 February 2019; accepted 26 March 2019; published online 11 June 2019

The propagation of $\mathrm{SH}$ wave in a heterogeneous initially stressed viscoelastic layer lying over a heterogeneous initially stressed orthotropic half-space due to a point source is analysed mathematically. The dispersion equation of SH wave is obtained for the propagation of SH wave in a specified model. The method of Green's function and Fourier transformation is incorporated to obtain the dispersion equation. The curves of dispersion equation are sketched for various values of heterogeneous parameters and initial stress on angular frequency, phase velocity and damping velocity in respect of wave number. The dispersion equation is derived for some special cases which reduces to the classical equation of Lovetype wave. The present study reveals the effect of heterogeneous parameter and initial stress associated with both viscoelastic and orthotropic media.

Keywords. Point source; Green's function; fourier transformation; viscoelastic; orthotropic; initial stress.

\section{Introduction}

The propagation of seismic waves in every heterogeneous layer is appreciable in the field of earthquake engineering and seismology because of the existence of heterogeneity which lies in the Earth's crust. Seismic study reflects that the Earth contains various layers having different densities and rigidities. Especially, at the time of sedimentation process and different geotechnical processes, it makes the interior of the Earth to be heterogeneous and anisotropic. Green's function technique is utilised to solve of a heterogeneous linear differential equation, defined on a domain, with specified initial conditions or boundary conditions. The nature of Earth's media is dispersive and attenuating with the propagation of mechanical waves.
Orthotropic material is a fragment of an anisotropic materials because their characteristics vary when quantified from various directions. The Earth is assumed to be an initially stressed medium consisting of different heterogeneous crustal layers. A huge volume of initial stress occurs in a medium because of different physical structures. Keeping this in mind, it must be relevant to study the propagation of elastic waves assuming the medium to be a initially stressed medium. Kundu et al. (2015) studied the propagation of SH wave in an orthotropic medium sandwiched between a homogeneous and non-homogeneous media. Daley and Krebes (2004) examined that simple reflected and transmitted particle displacements occur because of point torque source at the surface in a viscoelastic medium and a half-space. Chatterjee and 
Table 1. Phase velocity of SH wave for particular cases as given in figure 23.

\begin{tabular}{lcccccccccc}
\hline Figure 23 & $k_{01} H$ & 0.002092 & Error & 0.3522 & Error & 0.5689 & Error & 0.9149 & Error \\
\hline Phase velocity & Fixed curve & 0.5984 & & 0.6000 & & 0.5855 & & 0.2024 \\
& Case 1 & 0.6064 & 1.33 & 0.608 & 1.33 & 0.5952 & 1.65 & 0.2008 & 0.79 \\
& Case 2 & 0.6897 & 15.25 & 0.6785 & 13.08 & 0.5871 & 0.27 & 0 & 100 \\
& Case 3 & 0.6785 & 13.38 & 0.6673 & 11.21 & 0.592 & 1.11 & 0 & 100 & 0 \\
& Case 4 & 0.5791 & 3.22 & 0.5599 & 6.68 & 0.3194 & 45.44 & 0 & 100 \\
& Average error in percentage & & 8.295 & & 8.075 & & 12.11 & 75.197 \\
\hline
\end{tabular}

Chattopadhyay (2015) studied the propagation of shear waves in a viscoelastic heterogenous medium. Kundu et al. (2016) deal with the effect of point source on the propagation of Love wave in a heterogeneous layer and inhomogeneous half-space. Chattopadhyay et al. (2010) studied the propagation of $\mathrm{SH}$ wave in an irregular viscoelastic layer overlying a semi-infinite half-space. The propagation of $\mathrm{SH}$ wave in a viscoelastic heterogeneous layer lying over a half-space having self-weight is discussed by Sahu et al. (2014).

The blemish of perceptibility of materials such as metals, concrete, soil and mud cannot be disregarded in the Earth's crust. When seismic waves propagate through the Earth's interior, they are conceived not only by anisotropy but also by intrinsic viscosity of the media which was discussed by Carcione (1990). Recently, Kaur et al. (2017) studied the propagation of $\mathrm{SH}$ wave in a vertically heterogeneous viscoelastic layer lying over a micro-polar elastic half-space. Kakar and Kakar (2016) analysed the propagation of Love wave in a viscoelastic heterogeneous layer lying over a heterogeneous viscoelastic half-space. Transmission and reflection of plane $\mathrm{SH}$ wave through a perfectly conducting self-reinforced elastic layer sandwiched between two vertically inhomogeneous viscoelastic solid half-spaces is discussed by Chaudhary et al. (2005). The threedimensional scattering of near field for dilation and rotation in the time domain is studied by Sharma (2004).

Motivation behind considering viscoelastic material for the present study lies in the fact that the Earth's crust is composed of silicate and iron alloy materials. These materials respond nearly elastically under the application of small magnitude transient forces but viscously under the application of long duration forces. Materials such as coal, tar, sediments, etc., which are buried inside the Earth's surface, can be modelled as viscoelastic materials. Several authors have taken different form of variations, such as harmonic, linear, quadratic, for simulating the variation in density and other geological parameters inside the Earth.

A point source is a single identifiable localised source of some object. In our problem the source of disturbance is supposed at the interface of two distinct media. Because of occurrence of point source, the nature of wave may change.

In the present problem, the effect of point source on propagation of SH wave in a heterogeneous viscoelastic layer lying over a heterogeneous orthotropic half-space has been analysed mathematically as well as graphically which leads to new work. The research area of seismologists is the Earth's interior and its vibration applying mechanics of physics. With these viewpoints, this attempt will assist us to interpret the Earth's interior. The mathematical model considered in current work is an attempt which may better explain the phenomenon taking place in the crust during an earthquake. The present work ensures a mathematical model, which works like a pillar for field application in seismology (table 1).

\section{Geometrical conception of the problem}

The problem stands on the propagation of $\mathrm{SH}$ wave in a heterogeneous viscoelastic layer and a heterogeneous orthotropic half-space under the effect of point source. Both the media are supposed to be initially stressed. For the propagation of $\mathrm{SH}$ waves, it is assumed that the wave propagating along the $x$-axis and $z$-axis is pointing vertically downwards. The term $H$ symbolises for depth of the medium. The origin ' $O$ ' is situated at the surface of an upper viscoelastic medium. The source of disturbance $S$ is taken at the interface of these media as shown in figure 1 . The variation of heterogeneous parameters in upper viscoelastic layer is acknowledged in the following approach:

$$
\mu_{0}=\mu_{1} \mathrm{e}^{a z}, \mu_{0}^{\prime}=\mu_{1}^{\prime} \mathrm{e}^{a z}, \rho_{0}=\rho_{1} \mathrm{e}^{a z}, P_{0}=P_{1} \mathrm{e}^{a z},
$$




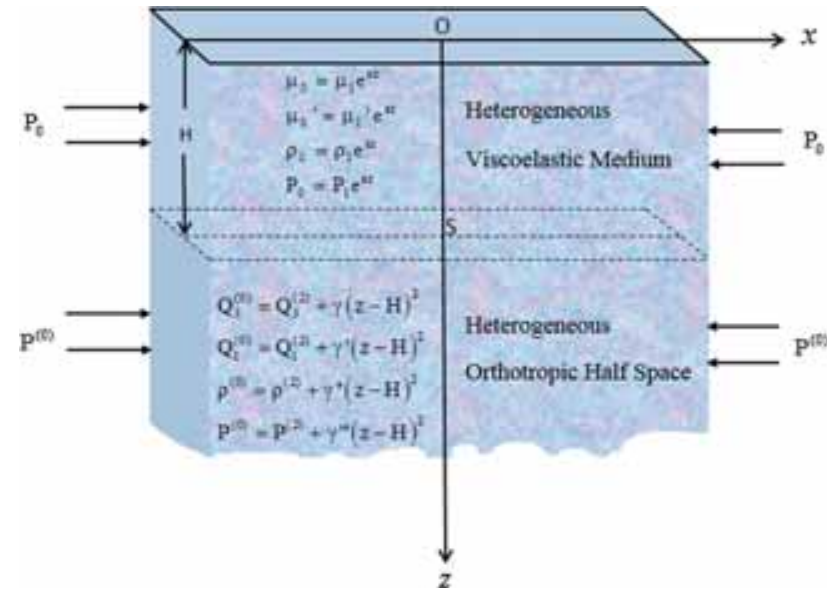

Figure 1. Geometry of the problem.

where $a$ is a constant having the dimension inverse of its length. The terms $\mu_{1}, \mu_{1}^{\prime}, \rho_{1}$ and $P_{1}$ symbolise for rigidity, viscosity, density and initial stress, respectively, while the terms $\mu_{0}, \mu_{0}^{\prime}, \rho_{0}$ and $P_{0}$ are heterogeneous rigidity, viscosity, density and initial stress that vary exponentially. The variation in shear modulus, density and initial stress of lower orthotropic half-space is contemplated in the following approach:

$$
\begin{aligned}
Q_{3}^{(0)} & =Q_{3}^{(2)}+\gamma(z-H)^{2}, \\
Q_{1}^{(0)} & =Q_{1}^{(2)}+\gamma^{\prime}(z-H)^{2}, \\
\rho^{(0)} & =\rho^{(2)}+\gamma^{\prime \prime}(z-H)^{2}, \\
P^{(0)} & =P^{(2)}+\gamma^{\prime \prime \prime}(z-H)^{2},
\end{aligned}
$$

where $\gamma, \gamma^{\prime}, \gamma^{\prime \prime}$ and $\gamma^{\prime \prime \prime}$ are considered to be small positive constants with suitable dimensions. The terms $Q_{1}^{(2)}$ and $Q_{3}^{(2)}$ are considered as shear modulus in an orthotropic medium. The terms $Q_{1}^{(2)}$, $Q_{3}^{(2)}, \rho^{(2)}$ and $P^{(2)}$ are fixed parameters while $Q_{1}^{(0)}$, $Q_{3}^{(0)}, \rho^{(0)}$ and $P^{(0)}$ are the functions of $z$.

\section{Solution for the upper viscoelastic layer}

A viscoelastic layer is supposed to be upper medium in the considered model. The governing equation of motion in a viscoelastic medium, because of source of disturbance, is expressed as (Chattopadhyay et al. 2010)

$$
\tau_{i j, j}+F_{i}=\rho_{0} \frac{\partial^{2} u_{i}}{\partial t^{2}}
$$

where $\tau_{i j}, \rho_{0}$ and $F_{i}$ correspond to the stress components, density and forces, respectively, at a point of the upper medium where elastic displacements are computed. The symbol $u_{i}\left(u_{1}, v_{1}, w_{1}\right)$ indicates the displacement components along the $x$-, $y$ - and $z$-axis, respectively. For $\mathrm{SH}$ wave propagation along the $x$-axis, it is assumed as

$$
u_{1}=w_{1}=0, \quad v_{1}=v_{1}(x, z, t) .
$$

In ponder of equation (4), the equation of motion for upper heterogeneous viscoelastic medium under the effect of point source is

$$
\begin{aligned}
& \frac{\partial}{\partial x}\left(\bar{\mu}_{0} \frac{\partial v_{1}}{\partial x}\right)+\frac{\partial}{\partial z}\left(\bar{\mu}_{0} \frac{\partial v_{1}}{\partial z}\right) \\
& \quad-P_{0} \frac{\partial \omega_{12}}{\partial x}-\rho_{0} \frac{\partial^{2} v_{1}}{\partial t^{2}}=4 \pi \sigma_{1}(r, t),
\end{aligned}
$$

where $\bar{\mu}_{0}=\mu_{0}+\mathrm{i} \omega \mu_{0}^{\prime} . \omega$ is the angular frequency and $\sigma_{1}(r, t)$ denotes the force density distribution in the upper medium due to point source. The symbol $r$ represents the distance from the origin, where the force is applied to a point at a time $t$. Substituting equation (1) into equation (5), it becomes

$$
\begin{gathered}
\frac{\partial^{2} v_{1}}{\partial x^{2}}+a \frac{\partial v_{1}}{\partial z}+\frac{\partial^{2} v_{1}}{\partial z^{2}}-\frac{\rho_{1}}{\bar{\mu}_{1}} \frac{\partial^{2} v_{1}}{\partial t^{2}} \\
-\frac{P_{1}}{2 \bar{\mu}_{1}} \frac{\partial^{2} v_{1}}{\partial x^{2}}=\frac{4 \pi \sigma_{1}(r, t) \mathrm{e}^{-a z}}{\bar{\mu}_{1}},
\end{gathered}
$$

where $\bar{\mu}_{1}=\mu_{1}+\mathrm{i} \omega \mu_{1}^{\prime}$ (say).

The following analytical solution may be proposed for the differential equation (6):

$$
\begin{aligned}
v_{1}(x, z, t) & =v_{1}(x, z) \mathrm{e}^{\mathrm{i} \omega t}, \\
\sigma_{1}(r, t) & =\sigma_{1}(r) \mathrm{e}^{\mathrm{i} \omega t} .
\end{aligned}
$$

Substituting equation (7) in equation (6), we arrive at the following equation:

$$
\begin{gathered}
\frac{\partial^{2} v_{1}}{\partial x^{2}}+a \frac{\partial v_{1}}{\partial z}+\frac{\partial^{2} v_{1}}{\partial z^{2}}+\frac{\rho_{1} \omega^{2}}{\bar{\mu}_{1}} v_{1} \\
-\frac{P_{1}}{2 \bar{\mu}_{1}} \frac{\partial^{2} v_{1}}{\partial x^{2}}=\frac{4 \pi \sigma_{1}(r) \mathrm{e}^{-a z}}{\bar{\mu}_{1}} .
\end{gathered}
$$

On complementing equation (8), the substitution is considered as follows:

$$
v_{1}(x, z)=V_{1}(x, z) \mathrm{e}^{-(a z / 2)},
$$

where $V_{i}(x, z), i=1,2$. The term $V_{i}(x, z)$ is a function of $x$ and $z$ that is used to express the displacement component $v_{i}(x, z)$ which will contribute to eliminate the first-order derivative and is 
helpful in solving the problem. Substituting equation (9) into equation (8), it is explicitly written as

$$
\begin{aligned}
& \left(1-\frac{P_{1}}{2 \bar{\mu}_{1}}\right) \frac{\partial^{2} V_{1}}{\partial x^{2}}+\frac{\partial^{2} V_{1}}{\partial z^{2}}+\left(\frac{\rho_{1} \omega^{2}}{\bar{\mu}_{1}}-\frac{a^{2}}{4}\right) V_{1} \\
& =\frac{4 \pi \sigma_{1}(r) \mathrm{e}^{-a z / 2}}{\bar{\mu}_{1}} .
\end{aligned}
$$

The disturbance caused by impulsive force $\sigma_{1}(r)$ may be symbolised in terms of the Dirac delta function at the source point as

$$
\sigma_{1}(r)=\delta(x) \delta(z-H) .
$$

Substituting equation (11) into equation (10), it is considered as

$$
\begin{aligned}
& \left(1-\frac{P_{1}}{2 \bar{\mu}_{1}}\right) \frac{\partial^{2} V_{1}}{\partial x^{2}}+\frac{\partial^{2} V_{1}}{\partial z^{2}}+\left(\frac{\rho_{1} \omega^{2}}{\bar{\mu}_{1}}-\frac{a^{2}}{4}\right) V_{1} \\
& =\frac{4 \pi \mathrm{e}^{-(a z / 2)}}{\bar{\mu}_{1}} \delta(x) \delta(z-H) .
\end{aligned}
$$

The Fourier transformation $\bar{V}_{1}(\chi, z)$ of $V_{1}(x, z)$ is defined as

$$
\bar{V}_{1}(\chi, z)=\frac{1}{2 \pi} \int_{-\infty}^{\infty} V_{1}(x, z) \mathrm{e}^{\mathrm{i} \chi x} \mathrm{~d} x
$$

Naturally, the inverse Fourier transformation $V_{1}(\chi, z)$ of $\bar{V}_{1}(x, z)$ is transformed as

$$
V_{1}(x, z)=\int_{-\infty}^{\infty} \bar{V}_{1}(\chi, z) \mathrm{e}^{-\mathrm{i} \chi x} \mathrm{~d} \chi .
$$

Now applying the Fourier transformation (13) in equation (12), it becomes

$$
\begin{aligned}
& \frac{\mathrm{d}^{2} \bar{V}_{1}}{\mathrm{~d} z^{2}}-\left(\chi^{2}\left(1-\frac{P_{1}}{2 \bar{\mu}_{1}}\right)-\frac{\rho_{1} \omega^{2}}{\bar{\mu}_{1}}+\frac{a^{2}}{4}\right) \bar{V}_{1} \\
& =\frac{2}{\bar{\mu}_{1}} \mathrm{e}^{-(a z / 2)} \delta(z-H) .
\end{aligned}
$$

Equation (15) can be replicated as

$$
\frac{\mathrm{d}^{2} \bar{V}_{1}}{\mathrm{~d} z^{2}}-\alpha^{2} \bar{V}_{1}=\frac{2}{\bar{\mu}_{1}} \mathrm{e}^{-(a z / 2)} \delta(z-H),
$$

where

$$
\begin{aligned}
\alpha^{2} & =\chi^{2}\left(1-\frac{P_{1}}{2 \bar{\mu}_{1}}\right)-k_{1}^{2}, \quad k_{1}^{2}=\frac{\rho_{1} \omega^{2}}{\bar{\mu}_{1}}-\frac{a^{2}}{4}, \\
\beta_{1} & =\sqrt{\frac{\mu_{1}}{\rho_{1}}} .
\end{aligned}
$$

Let $G^{(1)}\left(z / z_{0}\right)$ be the Green's function which satisfies the solution of upper media following the conditions $\mathrm{d} G^{(1)} / \mathrm{d} z=0$ at $z=0, H$, then equation (16) implies

$$
\frac{\mathrm{d}^{2} G^{(1)}\left(z / z_{0}\right)}{\mathrm{d} z^{2}}-\alpha^{2} G^{(1)}\left(z / z_{0}\right)=\delta\left(z-z_{0}\right) .
$$

Multiplying equation (17) by $\bar{V}_{1}$ and equation (16) by $G^{(1)}\left(z / z_{0}\right)$ and subtracting, it converts to

$$
\begin{aligned}
G^{(1)} & \left(z / z_{0}\right) \frac{\mathrm{d}^{2} \bar{V}_{1}}{\mathrm{~d} z^{2}}-\bar{V}_{1} \frac{\mathrm{d}^{2} G^{(1)}\left(z / z_{0}\right)}{\mathrm{d} z^{2}} \\
= & \frac{2}{\bar{\mu}_{1}} \mathrm{e}^{-(a z / 2)} \delta(z-H) G^{(1)}\left(z / z_{0}\right) \\
& -\delta\left(z-z_{0}\right) \bar{V}_{1}(z) .
\end{aligned}
$$

Integrating equation (18) with respect to $z$ having the limit from $z=0$ to $H$ (Vlaar 1966; Kundu et al. 2015 ), it reduces to

$$
\begin{aligned}
& G^{(1)}\left(H / z_{0}\right)\left[\frac{\mathrm{d} \bar{V}_{1}}{\mathrm{~d} z}\right]_{z=H} \\
& =\frac{2}{\bar{\mu}_{1}} \mathrm{e}^{-(a H / 2)} G^{(1)}\left(H / z_{0}\right)-\bar{V}_{1}\left(z_{0}\right) .
\end{aligned}
$$

Interchanging $z_{0}$ and $z$, and applying the symmetry property of Green's function in equation (19), it is expressed as

$$
\begin{aligned}
\bar{V}_{1}(z)= & \frac{2}{\bar{\mu}_{1}} \mathrm{e}^{-(a H / 2)} G^{(1)}(z / H) \\
& -G^{(1)}(z / H)\left[\frac{\mathrm{d} \bar{V}_{1}}{\mathrm{~d} z}\right]_{z=H} .
\end{aligned}
$$

\section{Dynamics for the heterogeneous orthotropic half-space}

The lower half-space is assumed to be constituted of an orthotropic material. The constitutive equation of motion neglecting the body force is expressed as follows (Biot 1962):

$$
\begin{aligned}
& \frac{\partial \kappa_{11}}{\partial x}+\frac{\partial \kappa_{12}}{\partial y}+\frac{\partial \kappa_{13}}{\partial z} \\
& -P^{(0)}\left(\frac{\partial \omega_{z}}{\partial y}-\frac{\partial \omega_{y}}{\partial z}\right)=\rho^{(0)} \frac{\partial^{2} w_{1}}{\partial t^{2}}, \\
& \frac{\partial \kappa_{21}}{\partial x}+\frac{\partial \kappa_{22}}{\partial y}+\frac{\partial \kappa_{23}}{\partial z}-P^{(0)} \frac{\partial \omega_{z}}{\partial x}=\rho^{(0)} \frac{\partial^{2} w_{2}}{\partial t^{2}}, \\
& \frac{\partial \kappa_{31}}{\partial x}+\frac{\partial \kappa_{32}}{\partial y}+\frac{\partial \kappa_{33}}{\partial z}+P^{(0)} \frac{\partial \omega_{y}}{\partial x}=\rho^{(0)} \frac{\partial^{2} w_{3}}{\partial t^{2}},
\end{aligned}
$$


where $w_{1}, w_{2}$ and $w_{3}$ are acknowledged as the components of displacement. The symbols $\kappa_{i j}$ $(i, j=1,2,3)$ and $\rho^{(0)}$ symbolise the incremental stress and density of the orthotropic half-space, respectively. The terms $\omega_{x}, \omega_{y}$ and $\omega_{z}$ denote the rotational components along the $x$-, $y$ - and $z$-axis, respectively. Stress and strain components of this medium are associated in such a manner that

$$
\begin{aligned}
& \kappa_{11}=C_{11} f_{11}+C_{22} f_{22}+C_{13} f_{33}, \kappa_{12}=2 Q_{3}^{(0)} f_{12}, \\
& \kappa_{22}=C_{21} f_{11}+C_{22} f_{22}+C_{23} f_{33}, \kappa_{23}=2 Q_{1}^{(0)} f_{23}, \\
& \kappa_{33}=C_{31} f_{11}+C_{32} f_{22}+C_{33} f_{33}, \kappa_{31}=2 Q_{2}^{(0)} f_{31},
\end{aligned}
$$

where $C_{i j}(i, j=1,2,3)$ and $Q_{i}^{(0)}(i=1,2,3)$ are used to signify the incremental normal elastic coefficients and shear modulus, respectively. The components of strain $f_{i j}$ are precisely written as

$$
f_{i j}=\frac{1}{2}\left(\frac{\partial w_{i}}{\partial x_{j}}+\frac{\partial w_{j}}{\partial x_{i}}\right),
$$

where $\left(x_{1}, x_{2}, x_{3}\right)=(x, y, z)$. Applying the conventional condition of SH wave, $w_{1}=0, w_{3}=0$ and $w_{2}=w_{2}(x, z, t)$ in equation (2), it novitiates to

$$
\begin{aligned}
& \frac{\partial}{\partial x}\left(\left(Q_{3}^{(0)}-\frac{P^{(0)}}{2}\right) \frac{\partial w_{2}}{\partial x}\right)+\frac{\partial}{\partial z}\left(Q_{1}^{(0)} \frac{\partial w_{2}}{\partial z}\right) \\
& \quad=\rho^{(0)} \frac{\partial^{2} w_{2}}{\partial t^{2}} .
\end{aligned}
$$

Equation (24) appears because the stress components $\kappa_{12}=2 Q_{1}^{(0)} f_{12}, \kappa_{23}=2 Q_{3}^{(0)} f_{23}$, and other stress components are zero. The equation of motion for lower orthotropic half-space under the influence of point source may be deliberated as

$$
\begin{aligned}
& \frac{\partial}{\partial x}\left(\left(Q_{3}^{(0)}-\frac{P^{(0)}}{2}\right) \frac{\partial w_{2}}{\partial x}\right)+\frac{\partial}{\partial z}\left(Q_{1}^{(0)} \frac{\partial w_{2}}{\partial z}\right) \\
& -\rho^{(0)} \frac{\partial^{2} w_{2}}{\partial t^{2}}=4 \pi \sigma_{2}(r, t),
\end{aligned}
$$

where $\sigma_{2}(r, t)$ exemplifies the force density distribution in the lower orthotropic half-space under the influence of source of disturbance. The term containing $r$ and $t$ in $\sigma_{2}(r, t)$ denotes the distance $r$ from the origin at a time $t$. The substitutions for $w_{2}$ and $\sigma_{2}$ are considered as

$$
\begin{aligned}
w_{2}(x, z, t) & =V_{2}(x, z) \mathrm{e}^{\mathrm{i} \omega t}, \\
\sigma_{2}(r, t) & =\sigma_{2}(r) \mathrm{e}^{\mathrm{i} \omega t},
\end{aligned}
$$

where $\omega=k c$ denotes the angular frequency, and $k$ and $c$ represent the wave number and wave velocity, respectively. At the source point, the disturbance occurs because of impulsive force $\sigma_{2}(r)$ which is expressed in the form of the Dirac delta function

$$
\sigma_{2}(r)=\delta(x) \delta(z-H) .
$$

Let us define the Fourier transformation $\bar{V}_{2}(\chi, z)$ of $V_{2}(x, z)$ as follows:

$$
\bar{V}_{2}(\chi, z)=\frac{1}{2 \pi} \int_{-\infty}^{\infty} V_{2}(x, z) \mathrm{e}^{\mathrm{i} \chi x} \mathrm{~d} x .
$$

Therefore, the inverse Fourier transformation $V_{2}(\chi, z)$ of $\bar{V}_{2}(x, z)$ takes the form

$$
V_{2}(x, z)=\int_{-\infty}^{\infty} \bar{V}_{2}(\chi, z) \mathrm{e}^{-\mathrm{i} \chi x} \mathrm{~d} \chi .
$$

Applying equations (26)-(28) in equation (25) with the help of equation (2), it is reduced to the following differential equation:

$$
\frac{\mathrm{d}^{2} \bar{V}_{2}}{\mathrm{~d} z^{2}}-\beta^{2} \bar{V}_{2}=4 \pi \sigma_{2}(z),
$$

where

$$
\beta^{2}=\frac{Q_{3}^{(2)}}{Q_{1}^{(2)}}\left(\chi^{2}-\frac{P_{2}^{(2)}}{2 Q_{3}^{(2)}}\right)-\frac{P^{(2)}}{Q_{1}^{(2)}} \frac{Q_{1}^{(2)}}{Q_{3}^{(2)}} \omega^{2}
$$

and

$$
\begin{aligned}
\beta_{2}^{2}=\frac{Q_{3}^{(2)}}{\rho^{(2)}} & , \\
4 \pi \sigma_{2}(z)= & -\frac{1}{Q_{1}^{(2)}}\left[\gamma^{\prime}(z-H)^{2} \frac{\mathrm{d}^{2} \bar{V}_{2}}{\mathrm{~d} z^{2}}\right. \\
& +2 \gamma^{\prime}(z-H) \frac{\mathrm{d} \bar{V}_{2}}{\mathrm{~d} z} \\
& +\left(\omega^{2} \gamma^{\prime}(z-H)^{2}-\gamma \chi^{2}(z-H)^{2}\right. \\
& \left.\left.+\chi^{2} \frac{\gamma^{\prime \prime \prime}}{2}(z-H)^{2}\right) \bar{V}_{2}\right] .
\end{aligned}
$$

Now, let us suppose that $G^{(2)}\left(z / z_{0}\right)$ be the Green's function which satisfies the solution of lower halfspace. Considering the case $\mathrm{d} G^{(2)} / \mathrm{d} z=0$ at $z=H$ and $\mathrm{d} G^{(2)} / \mathrm{d} z \rightarrow 0$ at $z \rightarrow-\infty$ (Kakar and Kakar 2016), then equation (30) transforms into

$$
\frac{\mathrm{d}^{2} G^{(2)}\left(z / z_{0}\right)}{\mathrm{d} z^{2}}-\beta^{2} G^{(2)}\left(z / z_{0}\right)=\delta\left(z-z_{0}\right) .
$$


Multiplying equation (32) by $\bar{V}_{2}$ and (30) by $G^{(2)}\left(z / z_{0}\right)$ then subtracting and integrating with respect to $z$ from $H$ to $\infty$, it becomes

$$
\begin{gathered}
\int_{H}^{\infty}\left(G^{(2)}\left(z / z_{0}\right) \frac{\mathrm{d}^{2} \bar{V}_{2}(z)}{\mathrm{d} z^{2}}-\bar{V}_{2}(z) \frac{\mathrm{d}^{2} G^{(2)}\left(z / z_{0}\right)}{\mathrm{d} z^{2}}\right) \mathrm{d} z \\
=\int_{H}^{\infty}\left(4 \pi \sigma_{2}(z) G^{(2)}\left(z / z_{0}\right)-\delta\left(z-z_{0}\right) \bar{V}_{2}(z)\right) \mathrm{d} z
\end{gathered}
$$

Integrating equation (33), it is converted into the following integral equation (Chattopadhyay et al. 2011; Kundu et al. 2016):

$$
\begin{aligned}
& -G^{(2)}\left(H / z_{0}\right)\left[\frac{\mathrm{d} \bar{V}_{2}}{\mathrm{~d} z}\right]_{z=H} \\
& =\int_{H}^{\infty}\left(4 \pi \sigma_{2}(z) G^{(2)}\left(z / z_{0}\right)\right) \mathrm{d} z-\bar{V}_{2}\left(z_{0}\right) \mathrm{d} z_{0} .
\end{aligned}
$$

Interchanging $z$ and $z_{0}$ in equation (34) and simplifying, it converts to the following equation:

$$
\begin{aligned}
\bar{V}_{2}(z)= & G^{(2)}(z / H)\left[\frac{\mathrm{d} \bar{V}_{2}}{\mathrm{~d} z}\right] \\
& +\int_{H}^{\infty} 4 \pi \sigma_{2}(z) G^{(2)}\left(z / z_{0}\right) \mathrm{d} z .
\end{aligned}
$$

\section{Boundary conditions}

The boundary conditions of present problem may be stated as follows:

1. At $z=0$, the upper surface is stress free which requires that

$$
\frac{\mathrm{d} \bar{V}_{1}}{\mathrm{~d} z}=0
$$

2. At $z=H$, the displacement is continuous, therefore it facilitates that

$$
\bar{V}_{1}=\bar{V}_{2}
$$

3. At $z=H$, the stress is continuous, therefore it manifests that

$$
\bar{\mu}_{1} \mathrm{e}^{a z} \frac{\mathrm{d} \bar{V}_{1}}{\mathrm{~d} z}=Q_{1}^{(2)} \frac{\mathrm{d} \bar{V}_{2}}{\mathrm{~d} z}
$$

From equations (20) and (35) and employing the second and third boundary conditions, it is expressed as

$$
\begin{aligned}
& \frac{2}{\bar{\mu}_{1}} \mathrm{e}^{-a H / 2} G^{(1)}(H / H)-G^{(1)}(H / H) \frac{Q_{1}^{(2)}}{\bar{\mu}_{1} \mathrm{e}^{a H}}\left[\frac{\mathrm{d} \bar{V}_{2}}{\mathrm{~d} z}\right] \\
& \quad=\left[G^{(2)}(H / H) \frac{\mathrm{d} \bar{V}_{2}}{\mathrm{~d} z}+\int_{H}^{\infty} 4 \pi \sigma_{2}(z) G^{(2)}\left(z / z_{0}\right) \mathrm{d} z\right] .
\end{aligned}
$$

From equation (39), it gives the value of $\mathrm{d} \bar{V}_{2} / \mathrm{d} z$ as follows:

$$
\begin{aligned}
& \frac{\mathrm{d} \bar{V}_{2}}{\mathrm{~d} z} \\
& =\frac{\frac{2}{\bar{\mu}_{1}} \mathrm{e}^{-a H / 2} G^{(1)}(H / H)-\int_{H}^{\infty} 4 \pi \sigma_{2}(z) G^{(2)}\left(z / z_{0}\right) \mathrm{d} z}{\left(G^{(2)}(H / H)+\frac{Q_{1}^{(2)} G^{(1)}(H / H)}{\bar{\mu}_{1} \mathrm{e}^{a H}}\right)} .
\end{aligned}
$$

Substituting equation (40) into equation (35), it transforms to

$$
\begin{aligned}
& \bar{V}_{2}(z)=G^{(2)}(z / H) \\
& {\left[\frac{\frac{2}{\bar{\mu}_{1}} \mathrm{e}^{-a H / 2} G^{(1)}(H / H)-\int_{H}^{\infty} 4 \pi \sigma_{2}(z) G^{(2)}\left(z / z_{0}\right) \mathrm{d} z}{\left(G^{(2)}(H / H)+\frac{Q_{1}^{(2)} G^{(1)}(H / H)}{\bar{\mu}_{1} \mathrm{e}^{a H}}\right)}\right]} \\
& \quad+\int_{H}^{\infty} 4 \pi \sigma_{2}(z) G^{(2)}\left(z / z_{0}\right) \mathrm{d} z .
\end{aligned}
$$

Taking the first-order approximation of equation (41), it becomes

$$
\bar{V}_{2}(z)=\frac{\frac{2}{\overline{\bar{\mu}}_{1}} \mathrm{e}^{-a H / 2} G^{(1)}(H / H) G^{(2)}(z / H)}{\left(G^{(2)}(H / H)+Q_{1}^{(2)} \frac{G^{(1)}(H / H)}{\bar{\mu}_{1} \mathrm{e}^{a H}}\right)} .
$$

From equations (20) and (35) and applying the second and third boundary conditions, we get

$$
\begin{gathered}
\frac{2}{\bar{\mu}_{1}} \mathrm{e}^{-a H / 2} G^{(1)}(H / H)-G^{(1)}(H / H)\left[\frac{\mathrm{d} \bar{V}_{1}}{\mathrm{~d} z}\right] \\
=\left[G^{(2)}(H / H) \frac{\bar{\mu}_{1} \mathrm{e}^{a H}}{Q_{1}^{(2)}}\left[\frac{\mathrm{d} \bar{V}_{1}}{\mathrm{~d} z}\right]\right. \\
\left.+\int_{H}^{\infty} 4 \pi \sigma_{2}(z) G_{2}\left(z / z_{0}\right) \mathrm{d} z\right] .
\end{gathered}
$$


Thus, we have

$$
\begin{aligned}
& \frac{\mathrm{d} \bar{V}_{1}}{\mathrm{~d} z} \\
& =\frac{\frac{2}{\bar{\mu}_{1}} \mathrm{e}^{-a H / 2} G^{(1)}(H / H)-\int_{H}^{\infty} 4 \pi \sigma_{2}(z) G^{(2)}\left(z / z_{0}\right) \mathrm{d} z}{G^{(1)}(H / H)+\frac{\bar{\mu}_{1}}{Q_{1}^{(2)}} \mathrm{e}^{a H} G^{(2)}(H / H)} .
\end{aligned}
$$

Substituting equation (44) into equation (20), it is represented as

$$
\begin{aligned}
\bar{V}_{1}(z)= & \frac{2 \mathrm{e}^{a H / 2} G^{(1)}(H / z) G^{(2)}(H / H)}{Q_{1}^{(2)} G^{(1)}(H / H)+\bar{\mu}_{1} \mathrm{e}^{a H} G^{(2)}(H / H)} \\
& -\frac{2 \mathrm{e}^{a H / 2} G^{(1)}(H / z) G^{(2)}(H / H)}{\left[Q_{1}^{(2)} G^{(1)}(H / H)+\bar{\mu}_{1} \mathrm{e}^{a H} G^{(2)}(H / H)\right]^{2}} \\
& \times \int_{H}^{\infty} X_{11} G^{(2)}\left(H / z_{0}\right) \mathrm{d} z_{0},
\end{aligned}
$$

where

$$
\begin{aligned}
X_{11}= & {\left[\gamma^{\prime}\left(z_{0}-H\right)^{2} \frac{\mathrm{d}^{2} G^{(2)}}{\mathrm{d} z^{2}}+2 \gamma^{\prime}\left(z_{0}-H\right) \frac{\mathrm{d} G^{(2)}}{\mathrm{d} z}\right.} \\
& +\left(\omega^{2}\left(z_{0}-H\right)^{2} \gamma^{\prime \prime}-\gamma \chi^{2}\left(z_{0}-H\right)^{2}\right. \\
& \left.\left.+\frac{\gamma^{\prime \prime \prime}}{2} \chi^{2}\left(z_{0}-H\right)^{2}\right) G^{(2)}\left(z_{0} / H\right)\right] .
\end{aligned}
$$

To acquire the solution, equation (16) can be considered as

$$
\frac{\mathrm{d}^{2} \theta}{\mathrm{d} z^{2}}-\alpha^{2} \theta=0
$$

Two independent solutions of equation (47) vanishing at $z=-\infty$ and $z=\infty$ are $\theta_{1}(z)=\mathrm{e}^{\alpha z}$ and $\theta_{2}(z)=\mathrm{e}^{-\alpha z}$. Therefore, the explication of equation (47) for a medium which is considered as

$$
\begin{aligned}
& \frac{\theta_{1}(z) \theta_{2}\left(z_{0}\right)}{W} \text { for } z<z_{0}, \\
& \frac{\theta_{1}\left(z_{0}\right) \theta_{2}(z)}{W} \text { for } z>z_{0},
\end{aligned}
$$

where $W=\theta_{1}\left(z_{0}\right) \theta_{2}^{\prime}(z)-\theta_{1}^{\prime}\left(z_{0}\right) \theta_{2}(z)=-2 \alpha \neq 0$. $W$ denotes the Wronskian of solution of differential equation (47). Therefore, the required solution is

$$
-\frac{\mathrm{e}^{-\alpha\left|z-z_{0}\right|}}{2 \alpha}
$$

The Green's function $G^{(1)}\left(z / z_{0}\right)$ satisfies the condition $\mathrm{d} G^{(1)} / \mathrm{d} z=0$ at $z=0$ and $z=H$.
Hence, the solution for upper layer in terms of Green's function is described as follows:

$$
G^{(1)}\left(z / z_{0}\right)=A_{1} \mathrm{e}^{\alpha z}+A_{2} \mathrm{e}^{-\alpha z}-\frac{\mathrm{e}^{-\alpha\left|z-z_{0}\right|}}{2 \alpha}
$$

where $A_{1}$ and $A_{2}$ are arbitrary constants that can be computed applying the condition $\mathrm{d} G^{(1)} / \mathrm{d} z=0$ at $z=0$ and $z=H$. Therefore, it leads to the following equation:

$$
\begin{aligned}
& G^{(1)}\left(z / z_{0}\right) \\
& =\frac{1}{2 \alpha}\left[\mathrm{e}^{-\alpha\left|z-z_{0}\right|}+\frac{\mathrm{e}^{\alpha z\left(\mathrm{e}^{-\alpha\left(H+z_{0}\right)}+\mathrm{e}^{-\alpha\left(H-z_{0}\right)}\right)}}{\mathrm{e}^{\alpha H}-\mathrm{e}^{-\alpha H}}\right. \\
& \left.\quad+\frac{\mathrm{e}^{-\alpha z\left(\mathrm{e}^{\alpha\left(H-z_{0}\right)}+\mathrm{e}^{-\alpha\left(H-z_{0}\right)}\right)}}{\mathrm{e}^{\alpha H}-\mathrm{e}^{-\alpha H}}\right]
\end{aligned}
$$

Thus, we have

$$
\begin{aligned}
G^{(1)}(H / z) & =-\frac{1}{\alpha}\left[\frac{\mathrm{e}^{\alpha z}+\mathrm{e}^{-\alpha z}}{\mathrm{e}^{\alpha H}-\mathrm{e}^{-\alpha H}}\right], \\
G^{(1)}(H / H) & =-\frac{1}{\alpha}\left[\frac{\mathrm{e}^{\alpha H}+\mathrm{e}^{-\alpha H}}{\mathrm{e}^{\alpha H}-\mathrm{e}^{-\alpha H}}\right], \\
G^{(2)}\left(z_{0} / H\right) & =-\frac{\mathrm{e}^{-\alpha\left(z_{0}-H\right)}}{\beta}, \\
G^{(2)}(H / H) & =-\frac{1}{\beta} .
\end{aligned}
$$

Using the approximation method, equation (51) and applying equations (52)-(55), the expression for $\bar{V}_{1}(z)$ is written as

$$
\begin{aligned}
& \bar{V}_{1}(z)=\frac{-2 \mathrm{e}^{a H / 2}\left(\mathrm{e}^{\alpha z}+\mathrm{e}^{-\alpha z}\right)}{Q_{1}^{(2)} \beta\left(\mathrm{e}^{\alpha H}+\mathrm{e}^{-\alpha H}\right)+\alpha \bar{\mu}_{1} \mathrm{e}^{a H}\left(\mathrm{e}^{\alpha H}-\mathrm{e}^{-\alpha H}\right)} \\
& \times\left[1+\frac{\left(\mathrm{e}^{\alpha H}+\mathrm{e}^{-\alpha H}\right)}{\left(\alpha \bar{\mu}_{1} \mathrm{e}^{a H}\left(\mathrm{e}^{\alpha H}-\mathrm{e}^{-\alpha H}\right)\right)+\beta Q_{1}^{(2)}\left(\mathrm{e}^{\alpha H}+\mathrm{e}^{-\alpha H}\right)}\right. \\
& \left.\quad \times X_{1}\right],
\end{aligned}
$$

where

$$
X_{1}=\frac{3 \gamma^{\prime} \alpha^{2}+\omega^{2} \gamma^{\prime \prime}-\gamma \chi^{2}+\frac{\gamma^{\prime \prime \prime}}{2} \chi^{2}}{4 \beta^{2} \alpha^{3}} .
$$

Neglecting the higher powers of $\gamma, \gamma^{\prime}, \gamma^{\prime \prime}$ and $\gamma^{\prime \prime \prime}$, it gives that 


$$
\begin{aligned}
& \bar{V}_{1}(z) \\
& =\frac{\frac{-2 \mathrm{e}^{\alpha H / 2}\left(\mathrm{e}^{\alpha z}+\mathrm{e}^{-\alpha z}\right)}{Q_{1}^{(2)} \beta\left(\mathrm{e}^{\alpha H}+\mathrm{e}^{-\alpha H}\right)+\mathrm{e}^{\alpha H} \alpha \bar{\mu}_{1}\left(\mathrm{e}^{\alpha H}-\mathrm{e}^{-\alpha H}\right)}}{\left[1-\frac{\left(\mathrm{e}^{\alpha H}+\mathrm{e}^{-\alpha H}\right)}{\mathrm{e}^{\alpha H}\left(\alpha \bar{\mu}_{1}\left(\mathrm{e}^{\alpha H}+\mathrm{e}^{-\alpha H}\right)\right)+\beta Q_{1}^{(2)}\left(\mathrm{e}^{\alpha H}-\mathrm{e}^{-\alpha H}\right)} \times X_{1}\right]} .
\end{aligned}
$$

Thus, $\bar{V}_{1}(z)$ may be rewritten as

$$
\bar{V}_{1}(z)=\frac{\frac{-2 \mathrm{e}^{a H / 2}\left(\mathrm{e}^{\alpha z}+\mathrm{e}^{-\alpha z}\right)}{M_{0}+N_{0}}}{1-\frac{\left(\mathrm{e}^{\alpha H}+\mathrm{e}^{-\alpha H}\right)}{\left(M_{0}+N_{0}\right)} X_{1}}
$$

when $M_{0}=\alpha \bar{\mu}_{1} \mathrm{e}^{a H}\left(\mathrm{e}^{\alpha H}+\mathrm{e}^{-\alpha H}\right)$ and $N_{0}=\beta\left(\mathrm{e}^{\alpha H}-\right.$ $\left.\mathrm{e}^{-\alpha H}\right) Q_{1}^{(2)}$.

Taking the inverse Fourier transformation of $\bar{V}_{1}(z)$, it becomes

$$
V_{1}(z)=-\int_{H}^{\infty} \frac{\mathrm{e}^{(-a / 2)(z-H)}\left(\mathrm{e}^{\alpha z}+\mathrm{e}^{-\alpha z}\right) \mathrm{e}^{-i \chi x \mathrm{~d} \chi}}{\left(M_{0}+N_{0}\right)-\left(\mathrm{e}^{\alpha H}+\mathrm{e}^{-\alpha H}\right) X_{1}} .
$$

The dispersion equation is obtained by making the denominator to be 0 , it gets

$$
\left(M_{0}+N_{0}\right)-\left(\mathrm{e}^{\alpha H}+\mathrm{e}^{-\alpha H}\right) X_{1}=0 .
$$

Equation (56) reduces to the dispersion equation which is expressed as follows:

$$
\tanh (\alpha H)=\frac{X_{1}-\beta Q_{1}^{(2)}}{\alpha \bar{\mu}_{1} \mathrm{e}^{a H}} .
$$

Assuming $k=k_{01}+k_{02}$ in equation (61) and then on equating real part we have

$$
\frac{\tanh \left(\alpha_{11} k_{01} H\right)\left(1+\tan ^{2}\left(\alpha_{12} k_{01} H\right)\right)}{1+\tan ^{2}\left(\alpha_{12} k_{01} H\right) \tanh ^{2}\left(\alpha_{11} k_{01} H\right)}=Y_{2}-Y_{5}
$$

and on equation imaginary part we get

$$
\frac{\tan \left(\alpha_{12} k_{01} H\right)\left(1-\tanh ^{2}\left(\alpha_{11} k_{01} H\right)\right)}{1+\tan ^{2}\left(\alpha_{12} k_{01} H\right) \tanh ^{2}\left(\alpha_{11} k_{01} H\right)}=Y_{3}-Y_{6} .
$$

When the wave number $k$ is assumed to be real, then equation (62) reduces to

$$
\frac{\tanh \left(\alpha_{11}^{\prime} k H\right)\left(1+\tan ^{2}\left(\alpha_{12}^{\prime} k H\right)\right)}{1+\tan ^{2}\left(\alpha_{12}^{\prime} k H\right) \tanh ^{2}\left(\alpha_{11}^{\prime} k H\right)}=Y_{2}^{\prime}-Y_{5}^{\prime} .
$$

When the wave number $k$ is assumed to be real, then equation (63) reduces to

$$
\frac{\tan \left(\alpha_{12}^{\prime} k H\right)\left(1-\tanh ^{2}\left(\alpha_{11}^{\prime} k H\right)\right)}{1+\tan ^{2}\left(\alpha_{12}^{\prime} k H\right) \tanh ^{2}\left(\alpha_{11}^{\prime} k H\right)}=Y_{3}^{\prime}-Y_{6}^{\prime} .
$$

The terms involved in the dispersion equation are given in appendices (see Appendices A1 and A2).

\section{Special cases}

Case I: When $\delta_{1} \rightarrow 0$ and $k_{11} \rightarrow 0$, i.e., viscosity is absent and wave number is real then dispersion equation (62) becomes

$$
\begin{aligned}
\tanh & {\left[\left(\sqrt{1-\frac{c^{2}}{\beta_{1}^{2}}}\right) k_{01} H\right] } \\
= & \frac{\left(1-\xi_{2}-\frac{c^{2}}{\beta_{2}^{2}}\right)\left(1-\xi_{1}-\frac{c^{2}}{\beta_{1}^{2}}\right) \frac{3 \gamma^{\prime} H^{4} Q_{1}^{(2)}}{4 Q_{1}^{(2)} \mu_{1}}}{\left(1-\xi_{1}-\frac{c^{2}}{\beta_{1}^{2}}\right)} \\
& -\frac{Q_{1}^{(2)}}{\mu_{1} \mathrm{e}^{a H}} \frac{\sqrt{1-\xi_{2}-\frac{c^{2}}{\beta_{2}^{2}}}}{1-\xi_{1}-\frac{c^{2}}{\beta_{1}^{2}}} .
\end{aligned}
$$

Equation (66) represents the dispersion equation of $\mathrm{SH}$ wave in a heterogeneous isotropic half-space under the initial stress over an initially stressed orthotropic half-space over the real.

Case II: When $P_{1} \rightarrow 0$ and $P_{2} \rightarrow 0$, i.e., medium and half-space both are assumed to be stress free, then equation (66) reduces to

$$
\begin{gathered}
\tanh \left[\left(\sqrt{1-\frac{c^{2}}{\beta_{1}^{2}}}\right) k_{01} H\right] \\
=\frac{\left(1-\frac{c^{2}}{\beta_{2}^{2}}\right)\left(1-\frac{c^{2}}{\beta_{1}^{2}}\right) \frac{3 \gamma^{\prime} H^{4} Q_{1}^{(2)}}{4 Q_{1}^{(2)} \mu_{1}}}{\left(1-\frac{c^{2}}{\beta_{1}^{2}}\right)} \\
-\frac{Q_{1}^{(2)}}{\mu_{1} \mathrm{e}^{a H}} \frac{\sqrt{1-\frac{c^{2}}{\beta_{2}^{2}}}}{\sqrt{1-\frac{c^{2}}{\beta_{1}^{2}}}}
\end{gathered}
$$

Equation (67) represents the dispersion equation of SH wave in a heterogeneous isotropic half-space over a heterogeneous orthotropic half-space over the real. 
Case III: When $\gamma^{\prime} \rightarrow$ 0, i.e., heterogeneity is absent in the layer, then equation (67) becomes

$$
\tanh \left[\left(\sqrt{1-\frac{c^{2}}{\beta_{1}^{2}}}\right) k_{01} H\right]=-\frac{Q_{1}^{(2)}}{\mu_{1} \mathrm{e}^{a H}} \frac{\sqrt{1-\frac{c^{2}}{\beta_{2}^{2}}}}{\sqrt{1-\frac{c^{2}}{\beta_{1}^{2}}}} .
$$

Equation (68) represents the dispersion equation of $\mathrm{SH}$ wave in an isotropic layer lying over a heterogeneous orthotropic half-space over the real.

Case $I V$ : When $a \rightarrow 0$ and $Q_{1}^{(2)}=\mu_{1}$ i.e., heterogeneity is absent in the half-space, then equation (68) becomes

$$
\tan \left[\left(\sqrt{\frac{c^{2}}{\beta_{1}^{2}}-1}\right) k_{01} H\right]=\frac{\sqrt{1-\frac{c^{2}}{\beta_{2}^{2}}}}{\sqrt{\frac{c^{2}}{\beta_{1}^{2}}-1}} .
$$

Equation (69) represents the dispersion equation of $\mathrm{SH}$ wave in an isotropic layer lying over an isotropic half-space. The dispersion equation agrees with the pre-established results from Kundu et al. (2016).

\section{Graphical observation}

For numerical discussion, we have taken the following relevant parameter in viscoelastic layer as (Caloi 1948; Romeo 2003)

$\rho_{1}=3321 \mathrm{~kg} / \mathrm{m}^{3}, \mu_{1}=7.1 \times 10^{10} \mathrm{~N} / \mathrm{m}^{2},\left(\mu_{1} / \mu_{1}^{\prime}\right)=$ $30 \mathrm{~s}^{-1}$.

For orthotropic semi-infinite half-space, the data has been taken from Gubbins (1990) as

$Q_{1}^{(2)}=5.82 \times 10^{10} \mathrm{~N} / \mathrm{m}^{2}, Q_{3}^{(2)}=3.99 \times 10^{10} \mathrm{~N} / \mathrm{m}^{2}$ and $\rho^{(2)}=4500 \mathrm{~kg} / \mathrm{m}^{3}$.

Figure 2 is drawn to show the effect of heterogeneous parameter $a / 2 k_{01}$ on phase velocity in respect of wave number. When the value of heterogeneous parameter increases, the phase velocity also increases. In respect of wave number and phase velocity, the phase velocity is constant up to wave number 0.65 then after that it decreases rapidly. The phase velocity is uniform for different values of heterogeneous parameter after the wave number 0.88 .

Figure 3 expresses the effect of $\xi_{1}=P_{1} / 2 \mu_{1}$ on phase velocity in respect of wave number. It is verified that as the value of $\xi_{1}$ increases, the phase velocity decreases. In respect of wave number and phase velocity, the phase velocity decreases very slightly as wave number increases up to wave number 0.5 and then decreases rapidly.

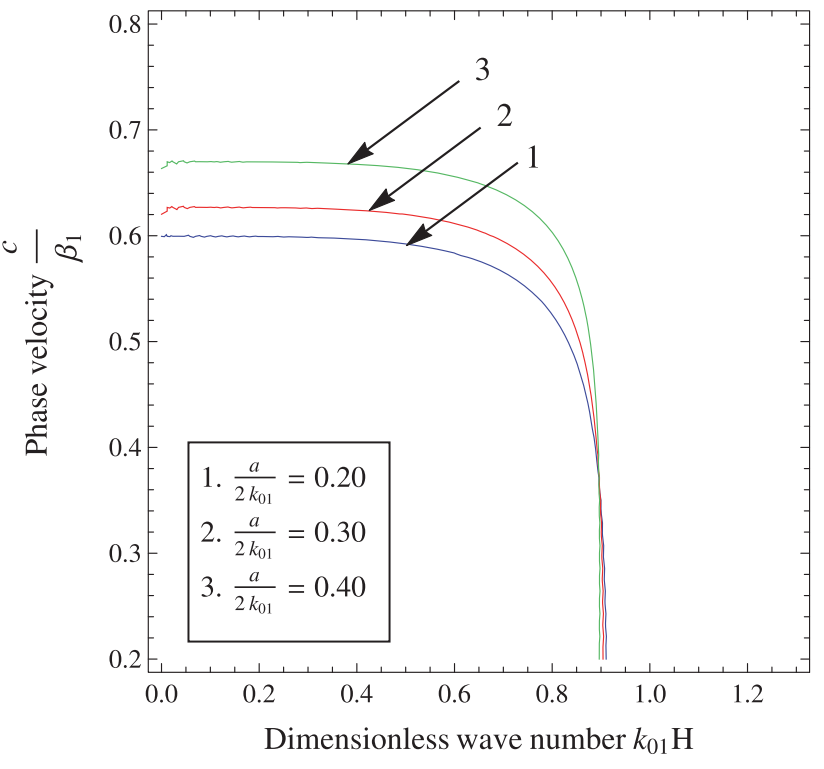

Figure 2. Variation of $a / 2 k_{01}$ with respect to phase velocity and dimensionless wave number.

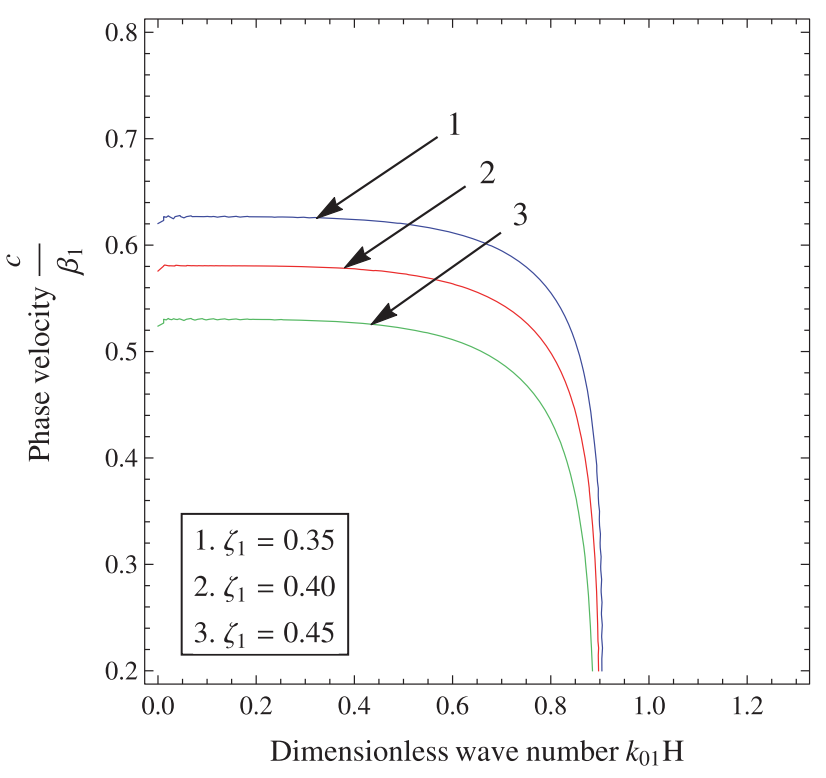

Figure 3. Variation of $\xi_{1}=P_{1} / 2 \mu_{1}$ with respect to phase velocity and dimensionless wave number.

Figure 4 examines the effect of initial stress of lower half-space on phase velocity in respect of wave number. When the value of $\xi_{2}=P_{2} / 2 Q_{3}^{(2)}$ increases, then the phase velocity also increases. In respect of wave number and phase velocity, the phase velocity decreases very slightly up to wave number 0.51 and then decreases rapidly.

Figure 5 examines the effect of heterogeneity associated with shear modulus of lower half-space. It is noticed that as the value of $\gamma^{\prime} H^{4} / 4 Q_{1}^{(2)}$ increases, the phase velocity also increases. In 


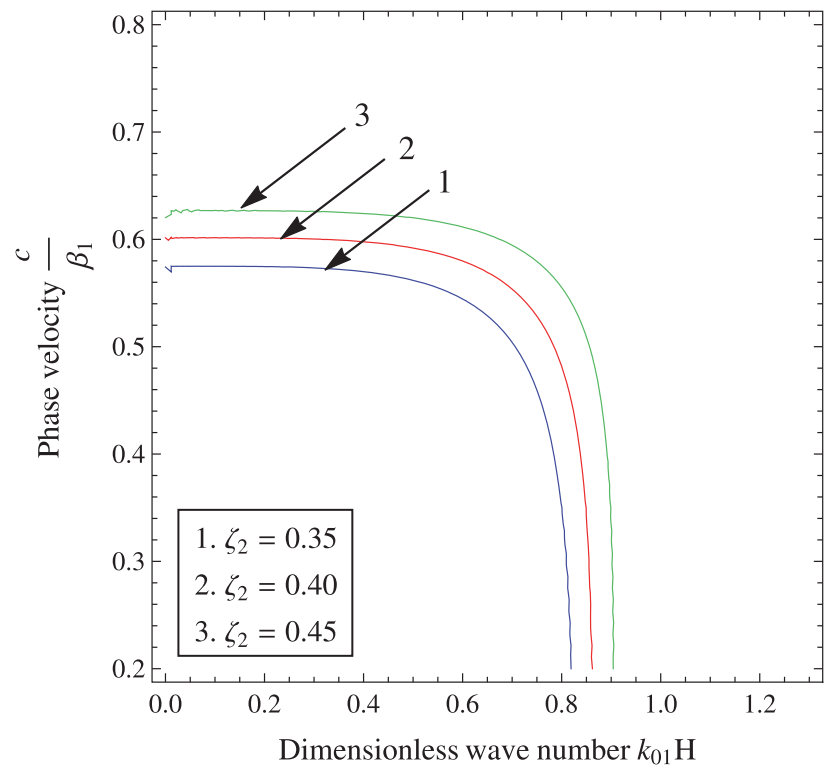

Figure 4. Variation of $\xi_{2}=P_{2} / 2 Q_{3}^{(2)}$ with respect to phase velocity and dimensionless wave number.

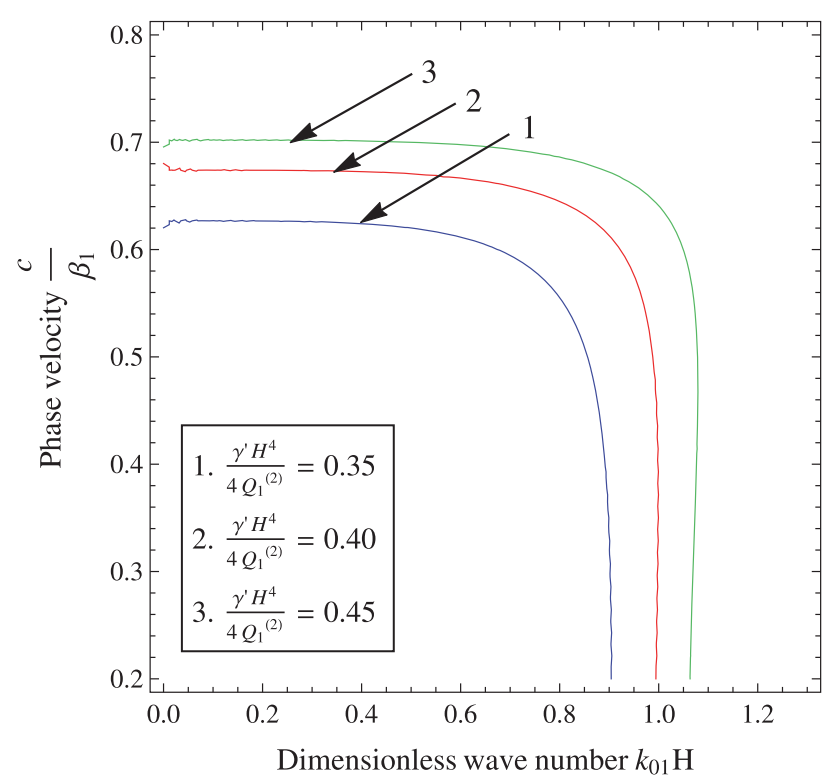

Figure 5. Variation of $\gamma^{\prime} H^{4} / 4 Q_{1}^{(2)}$ with respect to phase velocity and dimensionless wave number.

respect of wave number and phase velocity, the phase velocity decreases very slightly up to wave number 0.7 and then decreases rapidly.

Figure 6 manifests the effect of heterogeneity $\gamma^{\prime \prime} H^{4} / 4 \rho^{(2)}$ associated with density of the lower half-space on phase velocity in respect of dimensionless wave number. It is seen that as the value of heterogeneous parameter increases, the phase velocity also increases. In respect of wave number and phase velocity, the phase velocity decreases

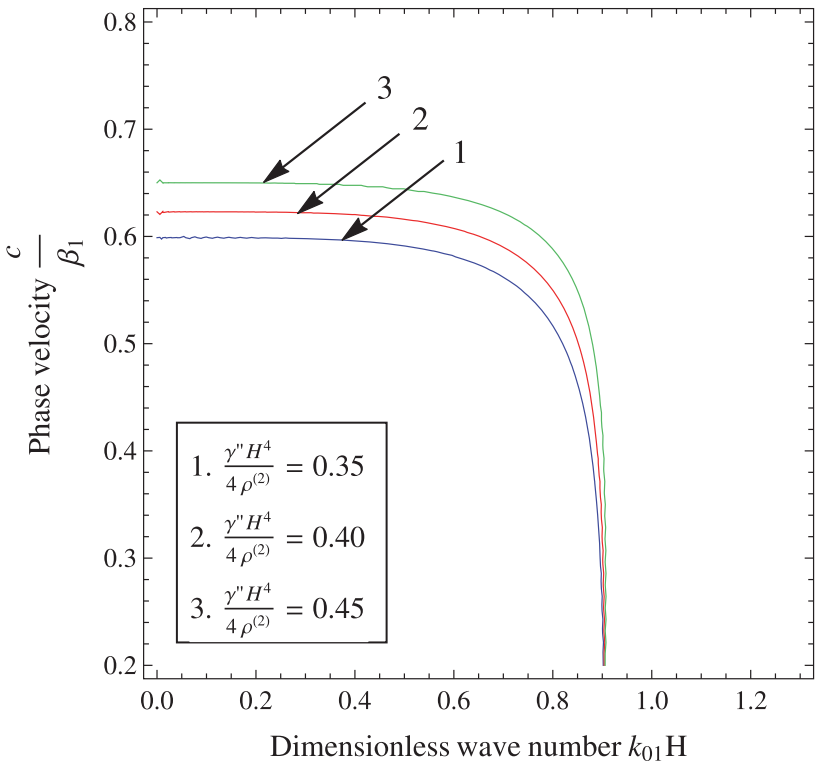

Figure 6. Variation of $\gamma^{\prime \prime} H^{4} / 4 \rho^{(2)}$ with respect to phase velocity and dimensionless wave number.

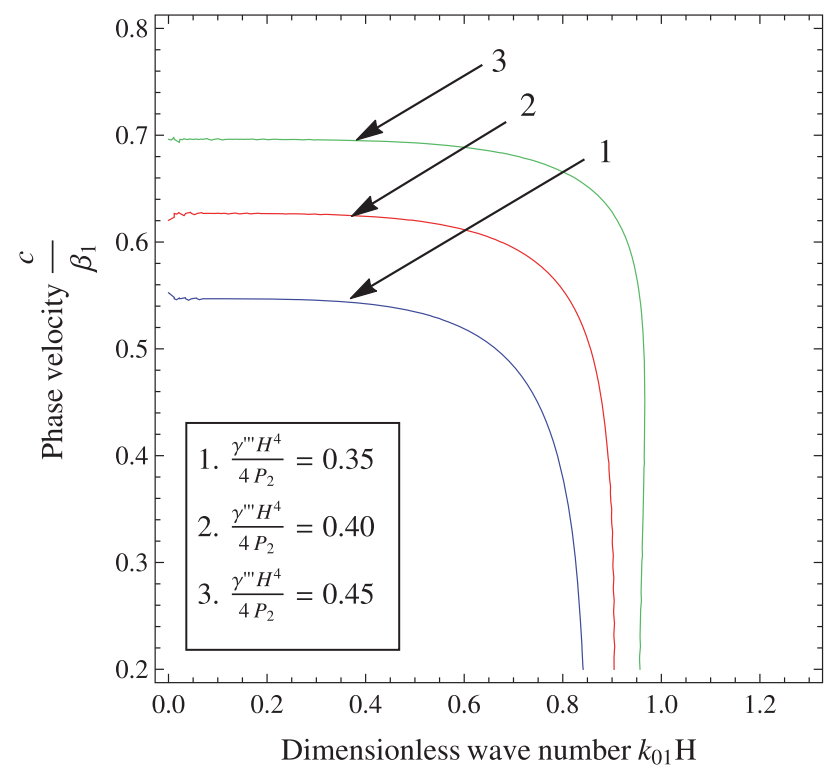

Figure 7. Variation of $\gamma^{\prime \prime \prime} H^{2} / 4 P_{2}$ with respect to phase velocity and dimensionless wave number.

slightly up to wave number 0.55 and then decreases rapidly.

Figure 7 shows the effect of heterogeneous parameter $\gamma^{\prime \prime \prime} H^{4} / 4 P_{2}$ associated with initial stress of lower half-space on phase velocity in respect of wave number. It is observed that when the value of heterogeneous parameter increases, the phase velocity also increases. In respect of wave number and phase velocity, the phase velocity decreases very slightly up to wave number 0.62 and then decreases rapidly. 


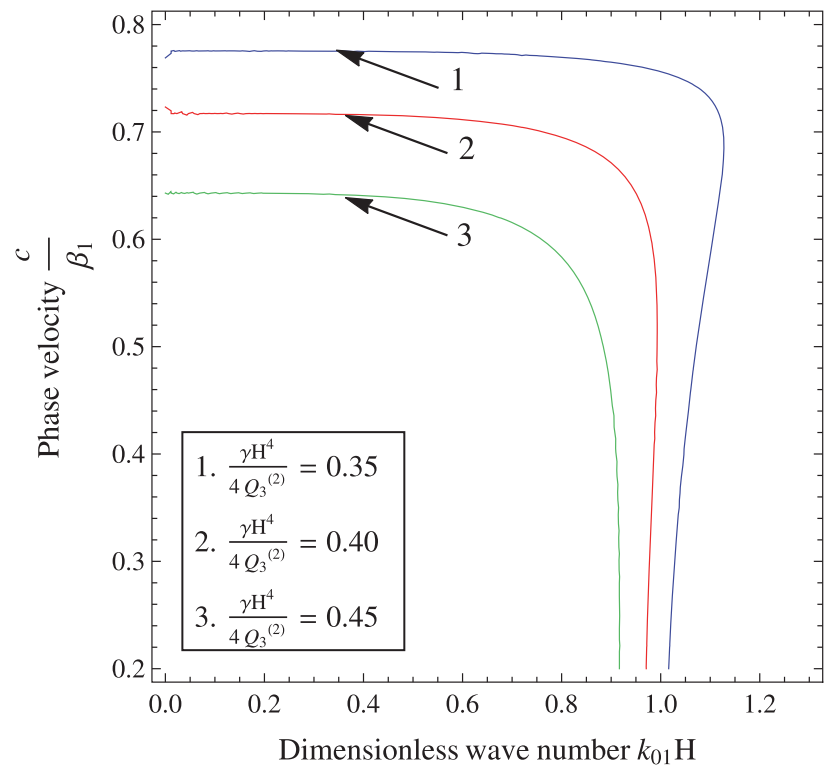

Figure 8. Variation of $\gamma H^{4} / 4 Q_{3}^{(2)}$ with respect to phase velocity and dimensionless wave number.

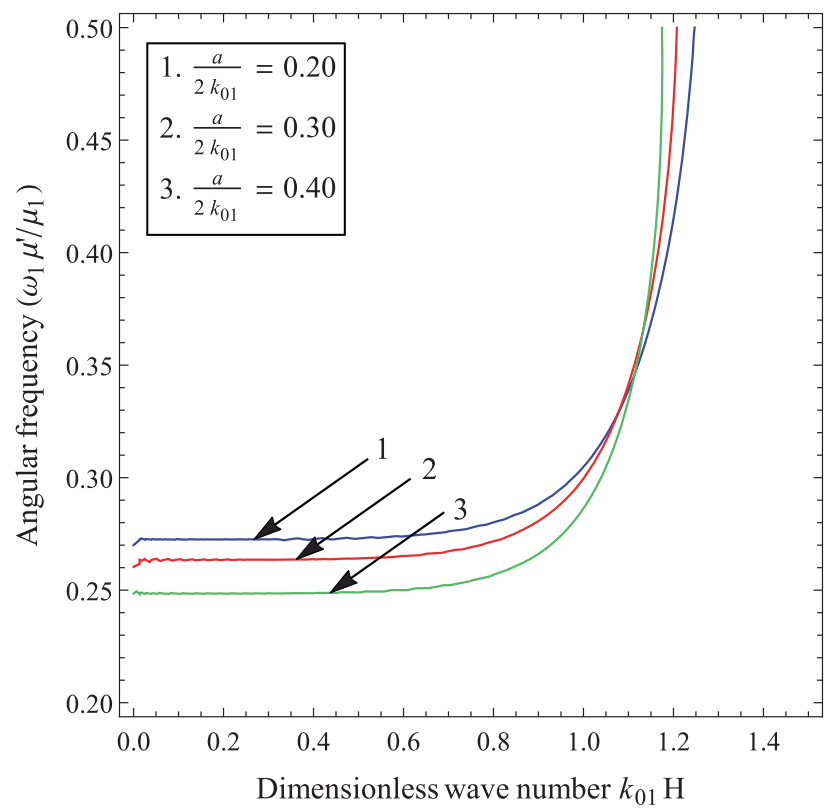

Figure 9 . Variation of $a / 2 k_{01}$ with respect to angular frequency and dimensionless wave number.

Figure 8 shows the effect of heterogeneity parameter $\gamma H^{4} / 4 Q_{3}^{(2)}$ associated with shear modulus of the orthotropic half-space on phase velocity in respect of wave number. It is analysed that the phase velocity decreases as heterogeneity parameter increases. In respect of wave number and phase velocity, the phase velocity decreases very slightly up to wave number 0.8 and then decreases rapidly.

Figure 9 examines the effect of heterogeneous parameter $a / 2 k_{01}$ on angular frequency in respect

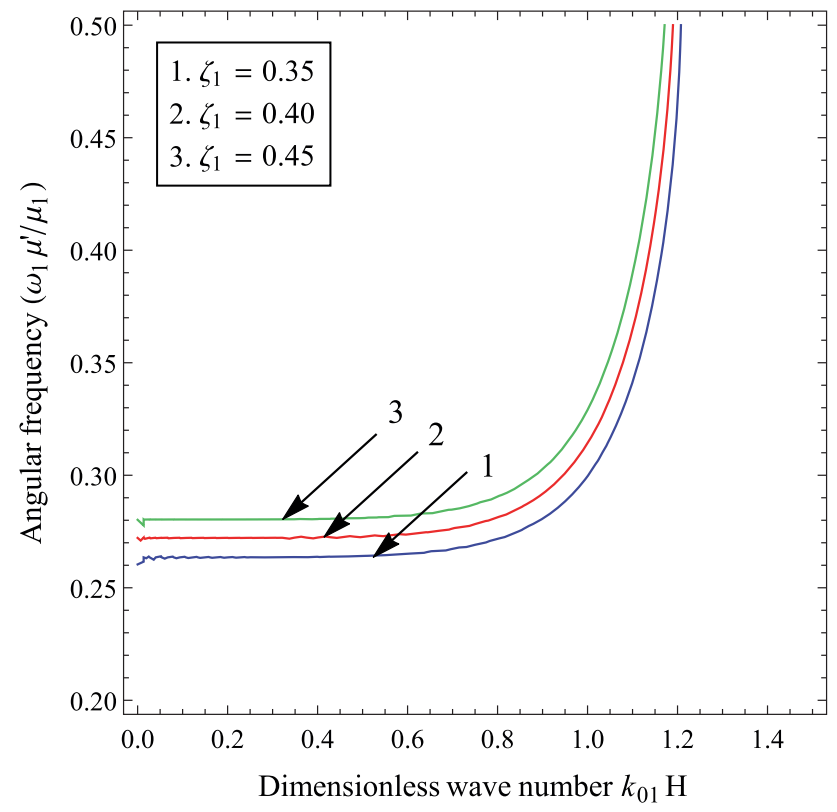

Figure 10. Variation of $\xi_{1}$ with respect to angular frequency and dimensionless wave number.

of wave number. It is noticed that when the value of $a / 2 k_{01}$ increases, the angular frequency decreases up to wave number 1.1 and then it behaves reversely. In respect of angular frequency and wave number, the angular frequency increases as the wave number increases.

Figure 10 expresses the effect of initial stress of medium on angular frequency in respect of wave number. It is seen that angular frequency increases as the wave number increases. In respect of wave number and angular frequency, the angular frequency increases as the wave number increases.

Figure 11 shows the effect of initial stress on angular frequency. It is examined that angular frequency decreases as initial stress increases. In respect of wave number and angular frequency, the angular frequency increases as the wave number increases.

Figure 12 analyses the effect of heterogeneity associated with shear modulus of the orthotropic half-space. It is expressed that as the value of $\gamma^{\prime \prime} H^{4} / 4 Q_{1}^{(2)}$ increases, the angular frequency decreases. In respect of angular frequency and wave number, the angular frequency increases as the wave number increases.

Figure 13 shows the effect of heterogeneous parameter associated with density of the lower halfspace. When the value of $\gamma^{\prime \prime} H^{4} / 4 \rho^{(2)}$ increases, the angular frequency decreases up to wave number 1.2 and then it behaves constantly. In respect of 


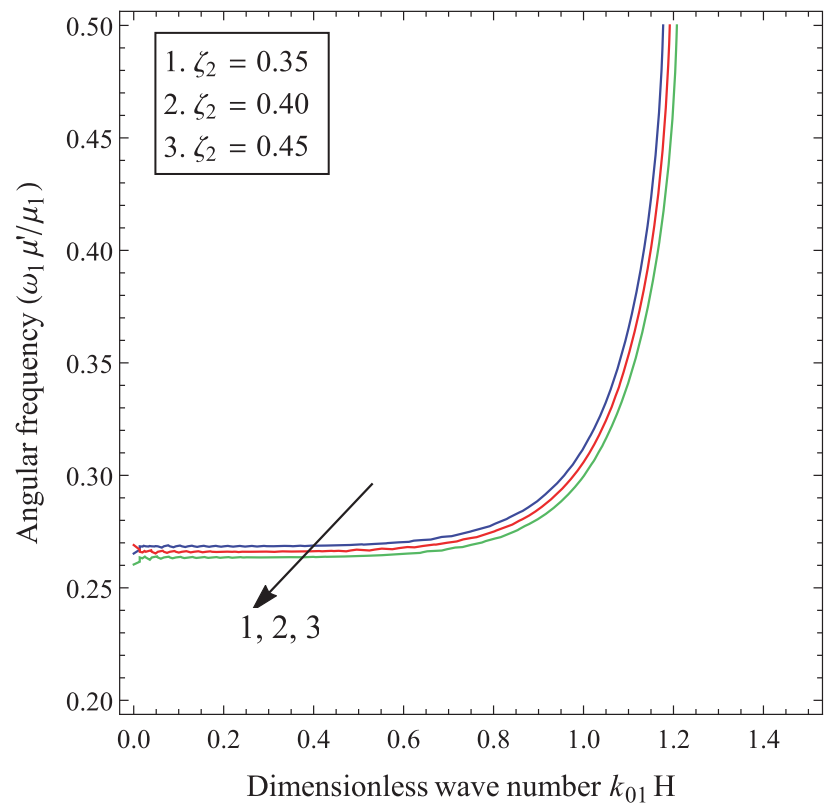

Figure 11. Variation of $\xi_{2}$ with respect to angular frequency and dimensionless wave number.

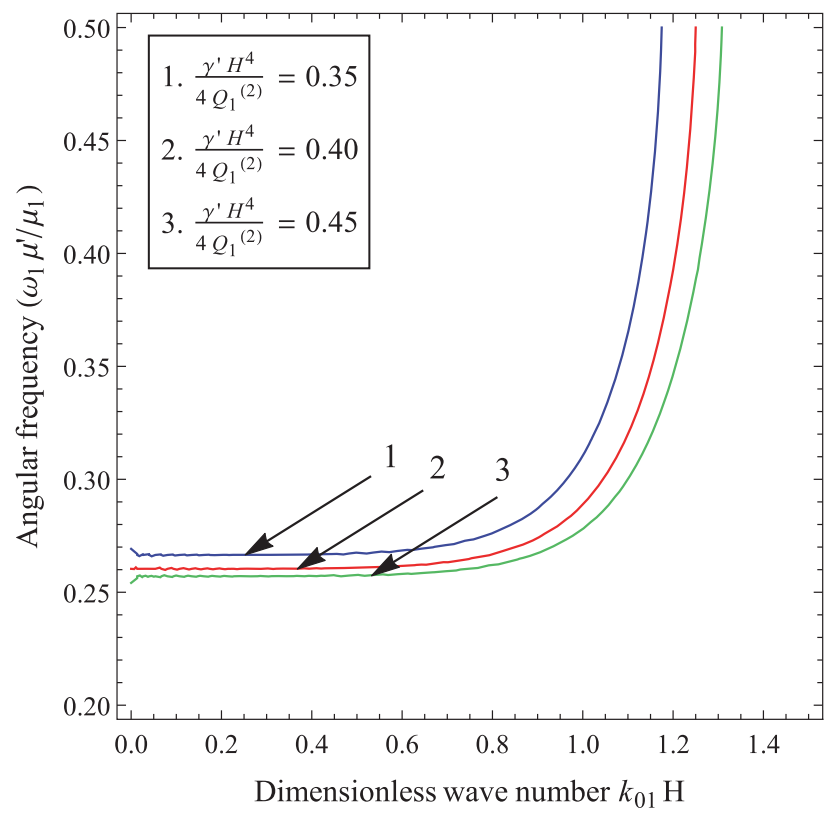

Figure 12. Variation of $\gamma^{\prime} H^{4} / 4 Q_{1}^{(2)}$ with respect to angular frequency and dimensionless wave number.

wave number and angular frequency, the angular frequency increases as the wave number increases.

Figure 14 shows the effect of heterogeneous parameter associated with initial stress of lower half-space. It is analysed that as the value of $\gamma^{\prime \prime} H^{4} / 4 \rho_{2}$ increases, the angular frequency decreases up to wave number 1.1 and then behaves reversely. In respect of wave number and angular frequency, the angular frequency increases as the wave number increases.

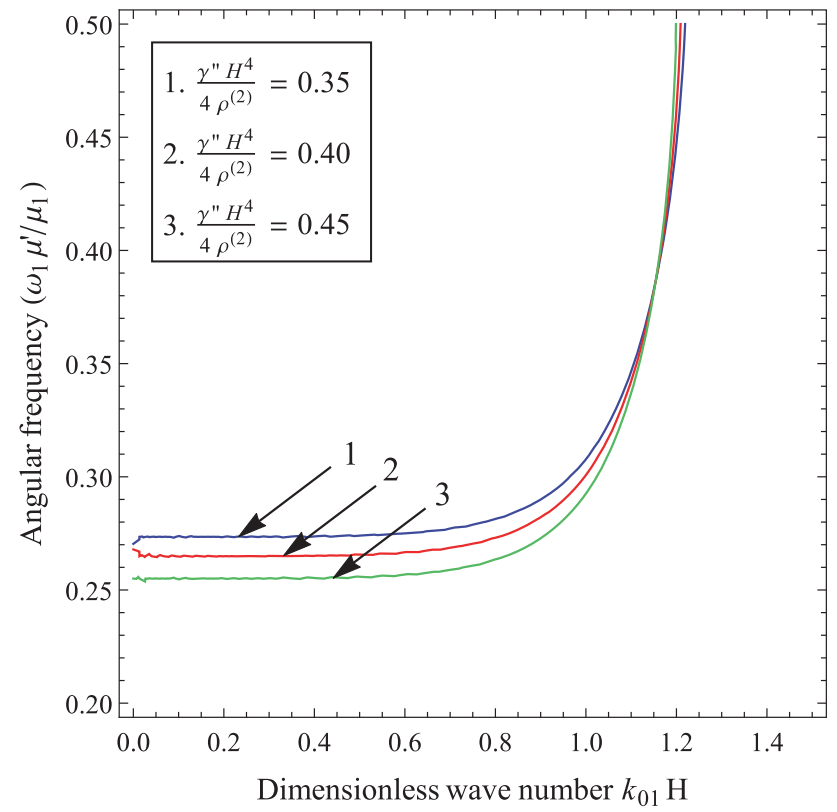

Figure 13. Variation of $\gamma^{\prime \prime} H^{4} / 4 \rho^{(2)}$ with respect to angular frequency and dimensionless wave number.

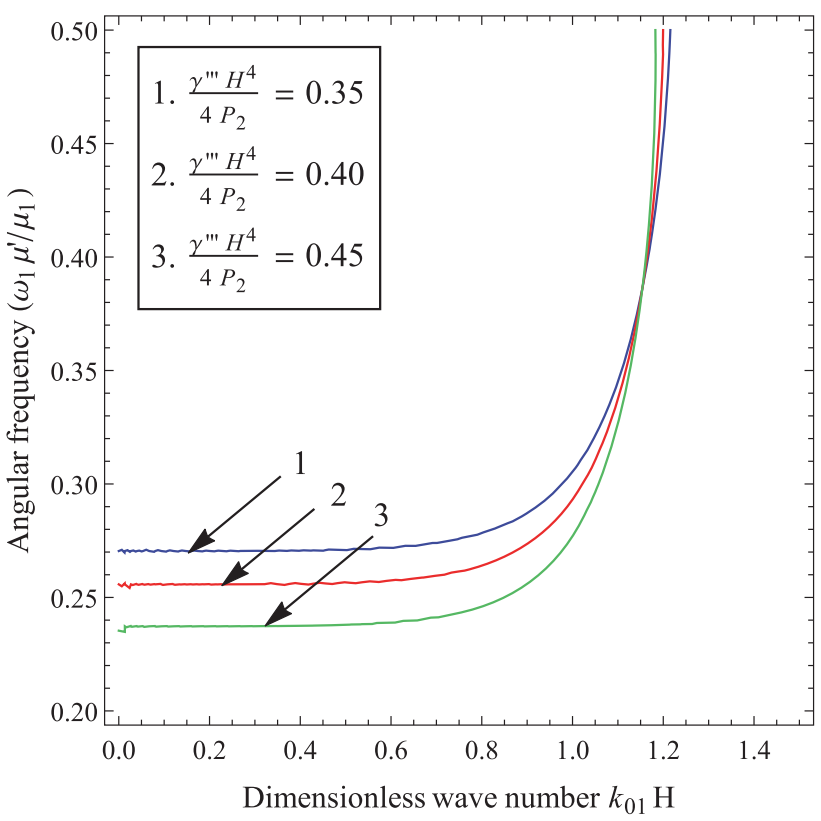

Figure 14. Variation of $\gamma^{\prime \prime \prime} H^{4} / 4 P_{2}$ with respect to angular frequency and dimensionless wave number.

Figure 15 shows the effect of heterogeneous parameter associated with shear modulus of lower half-space. When the value of $\gamma H^{4} / 4 Q_{3}^{(2)}$ increases, the angular frequency increases up to wave number 1.1 and then behaves reversely. In respect of wave number and angular frequency, the angular frequency increases as the wave number increases.

Figure 16 examines the effect of heterogeneous parameter $a / 2 k_{01}$ on damping velocity in respect 


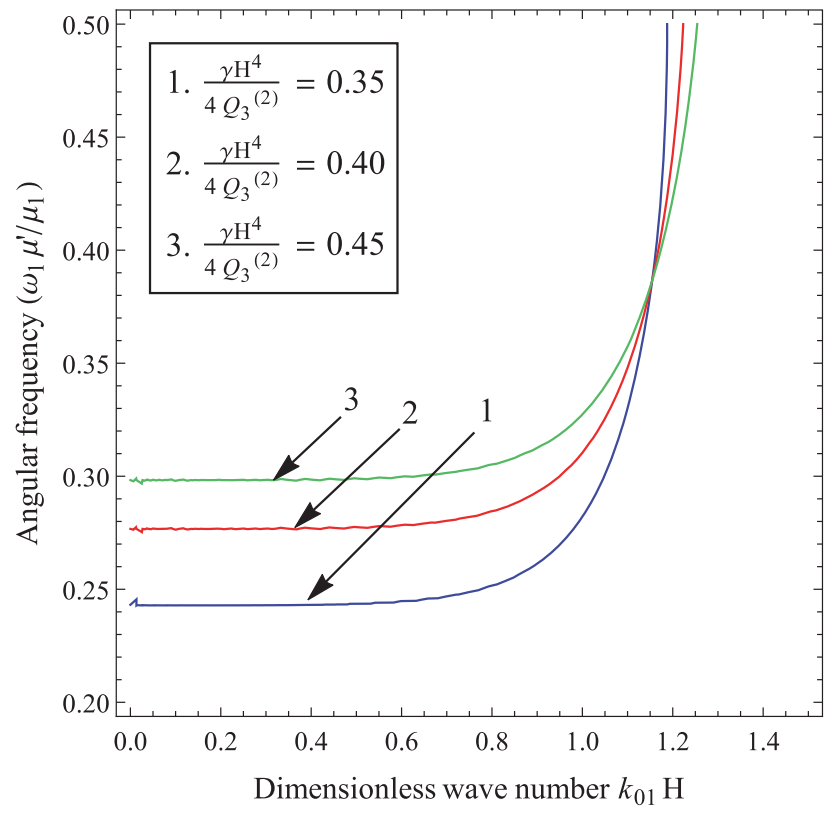

Figure 15. Variation of $\gamma H^{4} / 4 Q_{3}^{(2)}$ with respect to angular frequency and dimensionless wave number.

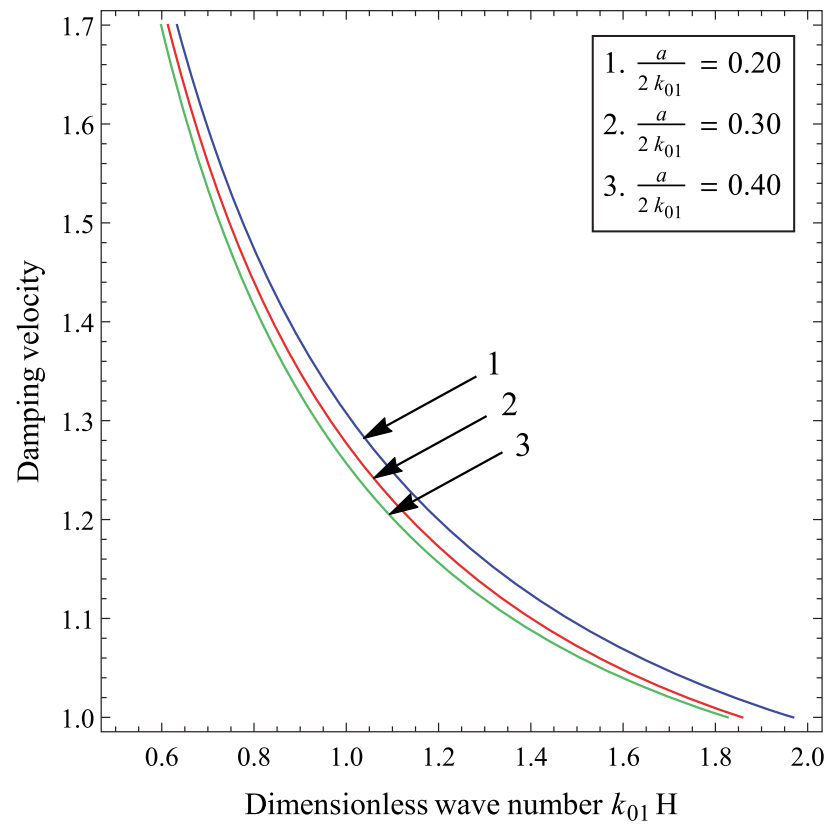

Figure 16. Variation of $a / 2 k_{01}$ with respect to damping velocity and dimensionless wave number.

of wave number. When the value of heterogeneous parameter $a / 2 k_{01}$ increases, the damping velocity decreases. In respect of wave number and damping velocity, the damping velocity decreases as the wave number increases.

Figure 17 expresses the effect of initial stress $\xi_{1}$ on damping velocity in respect of wave number. When the value of $\xi_{1}$ increases, the damping velocity increases. In respect of wave number and

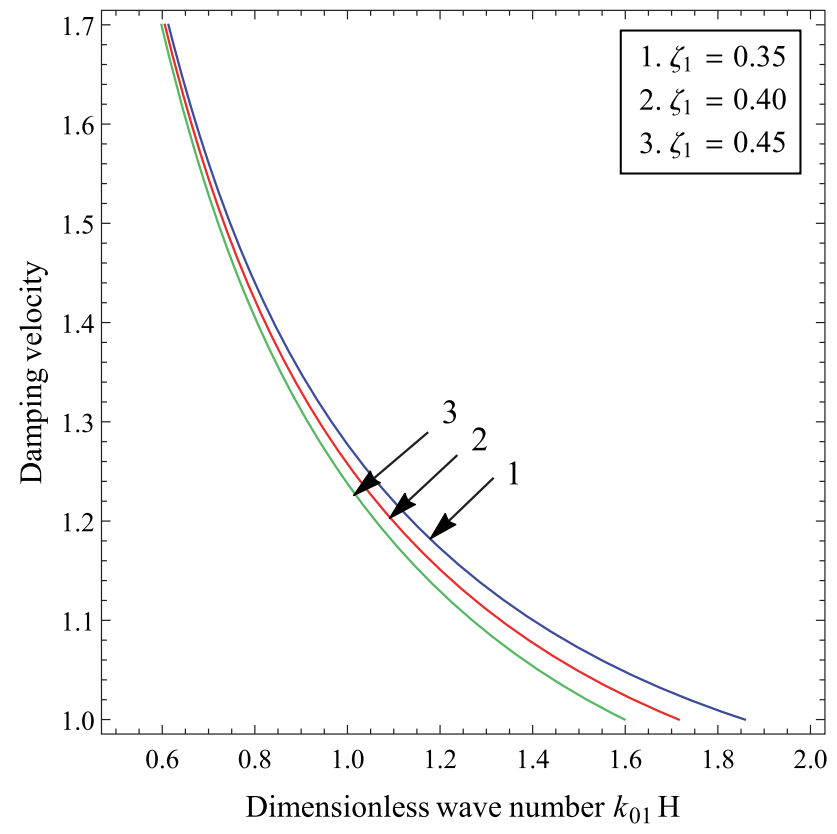

Figure 17. Variation of $\xi_{1}$ with respect to damping velocity and dimensionless wave number.

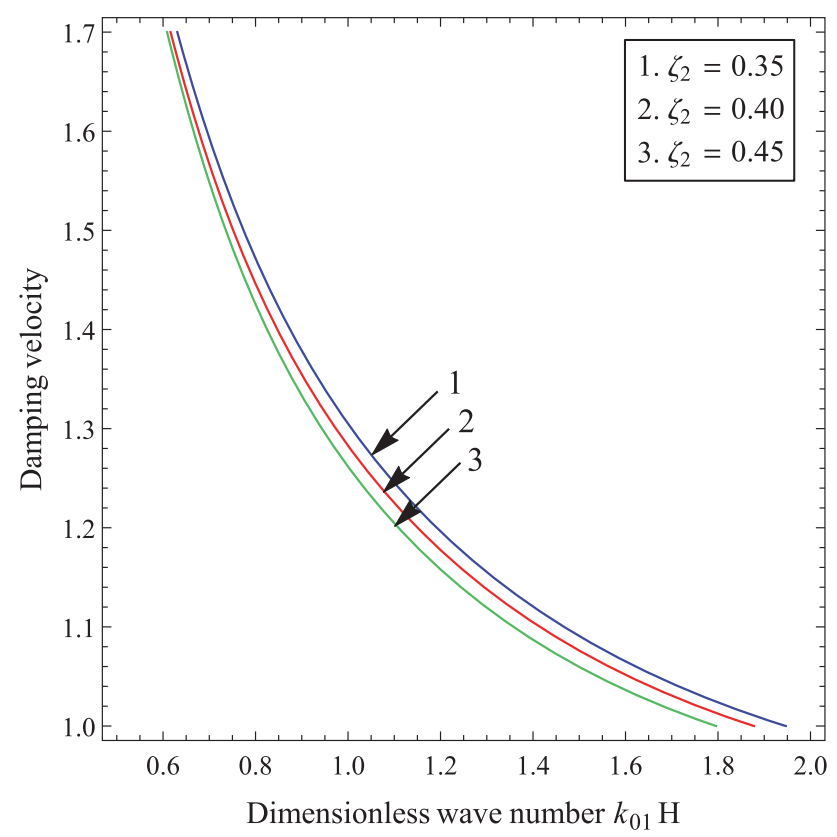

Figure 18. Variation of $\xi_{2}$ with respect to damping velocity and dimensionless wave number.

damping velocity, the damping velocity decreases as the wave number increases.

Figure 18 studies the effect of initial stress $\xi_{2}$ on damping velocity in respect of wave number. When the value of initial stress increases, the damping velocity decreases. In respect of wave number and damping velocity, the damping velocity decreases as the wave number increases. 


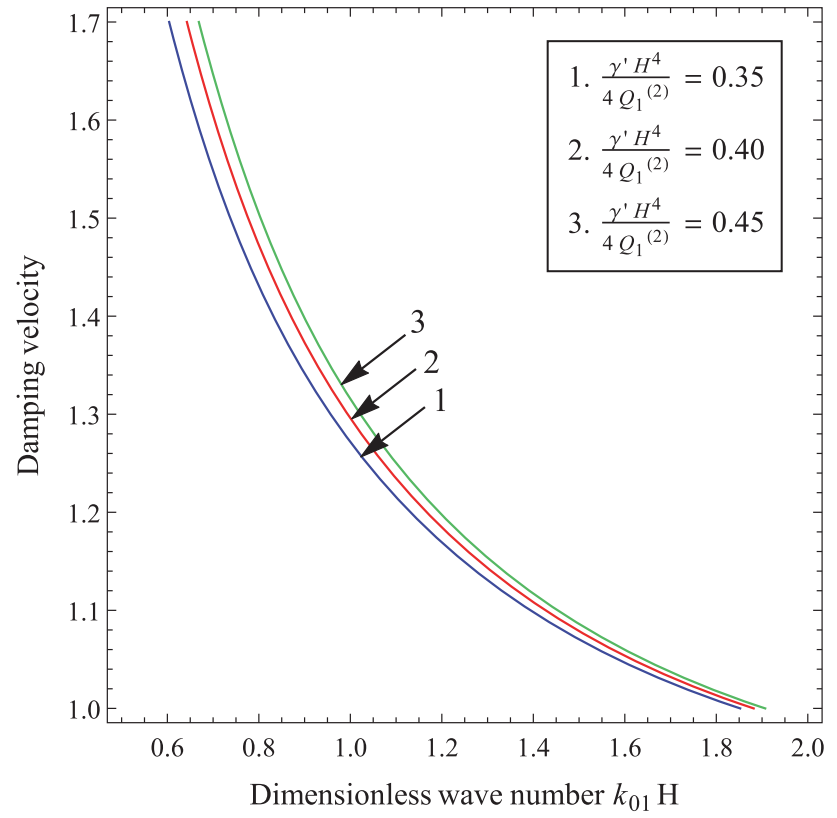

Figure 19. Variation of $\gamma^{\prime} H^{4} / 4 Q_{1}^{(2)}$ with respect to damping velocity and dimensionless wave number.

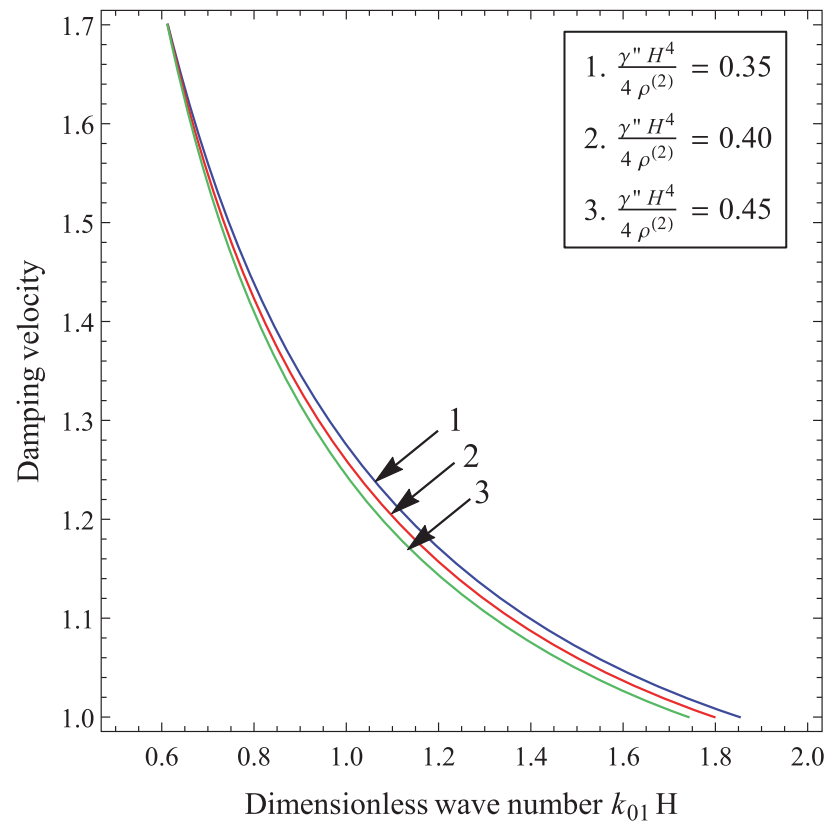

Figure 20. Variation of $\gamma^{\prime \prime} H^{4} / 4 \rho^{(2)}$ with respect to damping velocity and dimensionless wave number.

Figure 19 shows the effect of $\gamma^{\prime} H^{4} / 4 Q_{1}^{(2)}$ on damping velocity. When the value of $\gamma^{\prime} H^{4} / 4 Q_{1}^{(2)}$ increases, the damping velocity also increases. In respect of wave number and damping velocity, the damping velocity decreases as the wave number increases.

Figure 20 shows the effect of $\gamma^{\prime \prime} H^{4} / 4 \rho^{(2)}$ on damping velocity in respect of wave number. When the value of $\gamma^{\prime \prime} H^{4} / 4 \rho^{(2)}$ increases, the damping

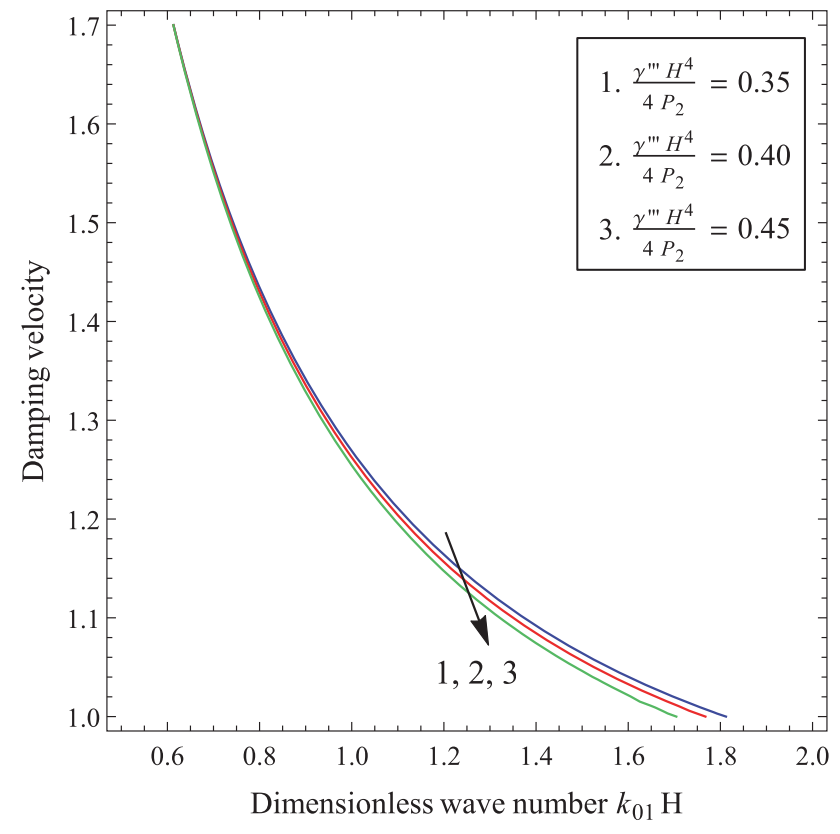

Figure 21. Variation of $\gamma^{\prime \prime \prime} H^{4} / 4 P_{2}$ with respect to damping velocity and dimensionless wave number.

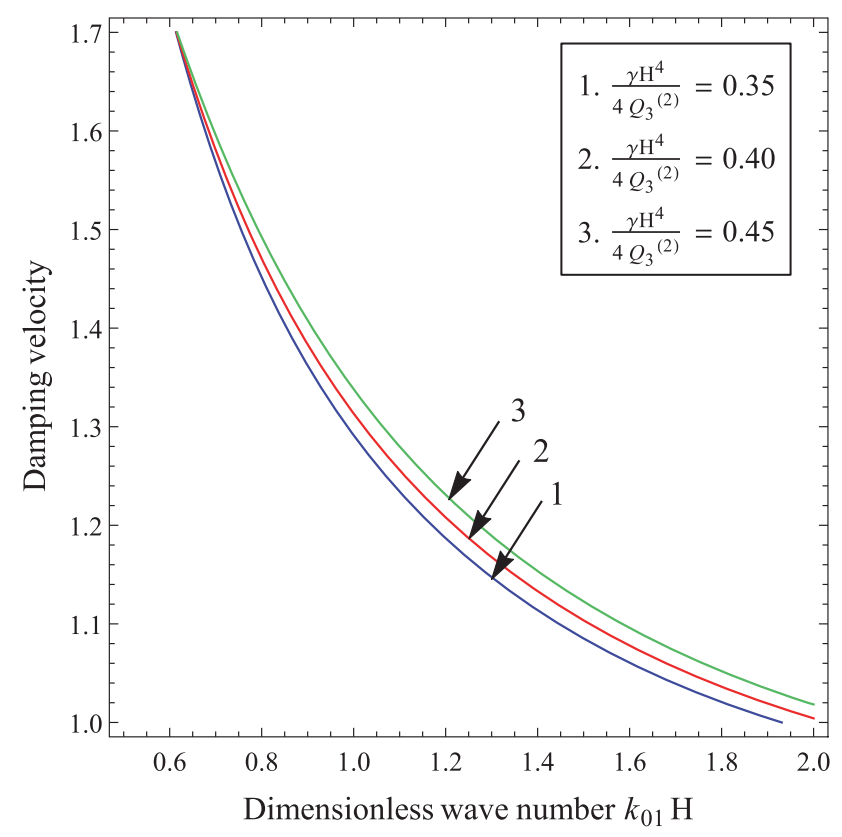

Figure 22. Variation of $\gamma H^{4} / 4 Q_{3}^{(2)}$ with respect to damping velocity and dimensionless wave number.

velocity decreases. In respect of wave number and damping velocity, the damping velocity decreases as the wave number increases.

Figure 21 expresses the effect of $\gamma^{\prime \prime} H^{4} / 4 P_{2}$ on damping velocity in respect of wave number. When the value of $\gamma^{\prime \prime} H^{4} / 4 P_{2}$ increases, the damping velocity decreases.

Figure 22 shows the effect of $\gamma H^{4} / 4 Q_{3}^{(2)}$ on damping velocity in respect of wave number. As 


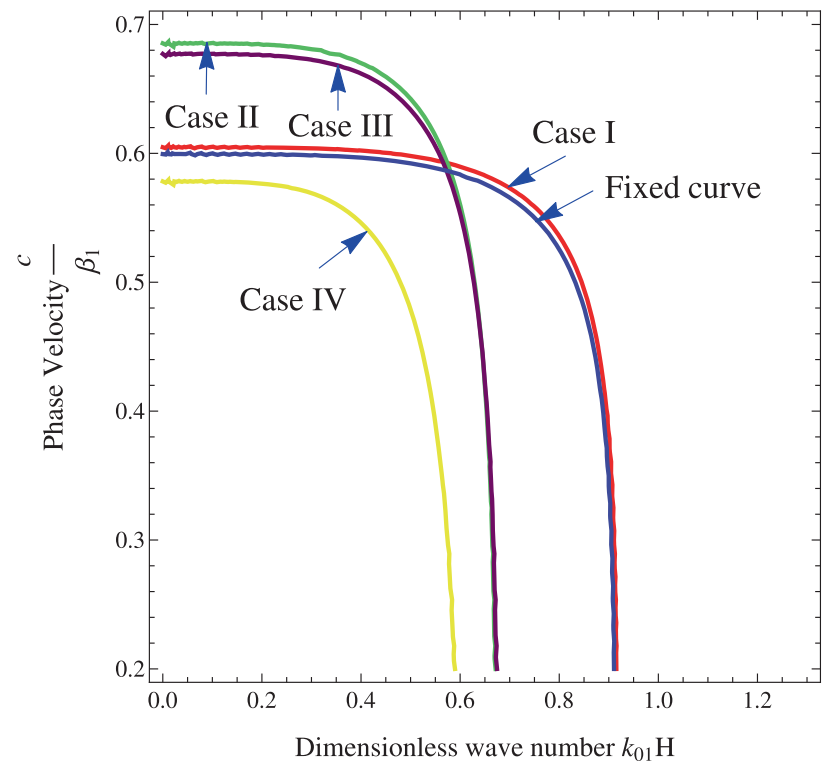

Figure 23. Particular cases of graphs with respect to phase velocity and dimensionless wave number.

the value of $\gamma^{\prime \prime} H^{4} / 4 Q_{3}^{(2)}$ increases, the damping velocity also increases. In respect of wave number and damping velocity, the damping velocity decreases as the wave number increases.

Figure 23 is drawn to show the particular cases separately. It is drawn in respect of dimensionless wave number and phase velocity. Curves 1,2,3,4 represent the particular cases $1,2,3,4$, respectively, while a fixed curve is drawn for all fixed values. Curve 4 represents the classical equation of Love-type wave. It is observed that phase velocity decreases when wave number increases.

\section{Error analysis}

This section determines the accuracy of propagation velocity of SH wave under graphical approach. In figure 23, five curves are drawn to show the effect of heterogeneous parameter associated with rigidity, density, viscosity and initial stress. A curve that is drawn for a fixed value, as taken in the numerical computation section, is presumed to be exact. An approximate error is calculated by the formula

$$
\left|\frac{\text { Exact value }- \text { Approximate value }}{\text { Exact value }} \times 100\right|
$$

(Vavryčuk 2008). A closed value of phase velocity is considered for the curve which is to be compared in respect of phase velocity of exact curve at every point. Applying the above technique, we have enumerated a table to estimate the error in graphs for all particular cases. The error analysis helps us to compare the theoretical work with the real-time data. Thus, it makes an important application for real data problem. We might mention that nickel, cobalt, chromium, aluminium, titanium and molybdenum are examples of viscoelastic materials.

\section{Conclusion}

The propagation of $\mathrm{SH}$ wave in a heterogeneous viscoelastic medium under initial stress lying over a heterogeneous orthotropic half-space has been studied under the effect of point source. The technique of Green's function is applied to find the dispersion equation and displacement component in the particular layer. From the above discussion, it can be concluded that

1. the dissipation factor, which is known as angular frequency, is a function of complex wave number;

2. the phase velocity decreases as the wave number increases;

3. the angular frequency increases when the wave number increases;

4. the heterogeneous parameters and initial stress have a significant effect on the propagation of $\mathrm{SH}$ wave influenced by point source;

5. the 'Mathematica' software is applied to study the effect of heterogeneous parameter associated with rigidity, density and internal friction of the layer.

This work is purely mathematical considering the geophysical phenomenon and it will behave like a bridge between geophysical application and mathematics certainly.

\section{Application}

Orthotropic materials are a subset of anisotropic materials. It is desirable to study the seismic waves in an anisotropic media, as the propagation of elastic waves in anisotropic media is fundamentally different from their propagation in isotropic media. As the Earth's crust and mantle are heterogeneous, it is interesting to know the propagation pattern of shear waves due to point source in heterogeneous media. Achenbach (2012) verified that the propagation of elastic surface wave is affected by 
the elastic properties of the medium through which they travel.

The present study has established that the increase in heterogeneity and viscoelastic parameter increases the phase velocity for viscoelastic media. The study of shear wave in this particular medium provides valuable information for selection of proper structural materials for the present day construction work. Earthquake source dynamics provides key elements for the prediction of ground motion, and to understand the physics of earthquake initiation, propagation and healing. The simplest possible model of seismic source is that of a point source buried in an elastic halfspace. A very important issue in seismology is the amount of energy radiated by seismic sources. The concepts of point source are widely applied in the field of oil exploration. It is also useful to remove the water pollution from an oil refinery waste water discharge outlet.

\section{Acknowledgements}

This research received no specific grant from any funding agency in the public, commercial or non-profit sectors.

\section{Appendix A1}

$$
\begin{aligned}
Y_{2}= & \left(\alpha_{15} X_{2}+\alpha_{16} X_{3}\right) X_{4}, \\
Y_{3}= & \left(\alpha_{15} X_{3}-\alpha_{16} X_{2}\right) X_{4}, X_{4}=\frac{1}{\left(k_{01} H\right)^{4} \mathrm{e}^{a H}}, \\
X_{2}= & 3 \frac{\gamma^{\prime} H^{4}}{4 Q_{1}^{(2)}} \frac{Q_{1}^{(2)}}{\mu_{1}}\left(\alpha_{11}^{2}-\alpha_{12}^{2}\right) \\
& +\frac{c^{2}}{\beta_{2}^{2}} \frac{Q_{3}^{(2)}}{\mu_{1}} \frac{\gamma^{\prime \prime} H^{4}}{4 \rho_{2}}\left(1-k_{11}^{2}\right) \\
& +\zeta_{2} \frac{Q_{3}^{(2)}}{\mu_{1}} \frac{\gamma^{\prime \prime \prime} H^{4}}{4 P_{2}}\left(1-k_{11}^{2}\right) \\
& -\frac{\gamma H^{4}}{4 Q_{3}^{(2)}} \frac{Q_{3}^{(2)}}{\mu_{1}}\left(1-k_{11}^{2}\right), \\
X_{3}= & 3 \frac{\gamma^{\prime} H^{4}}{4 Q_{1}^{(2)}} \frac{Q_{1}^{(2)}}{\mu_{1}}\left(2 \alpha_{11} \alpha_{12}\right)+2 k_{11} \frac{c^{2}}{\beta_{2}^{2}} \frac{Q_{3}^{(2)}}{\mu_{1}} \frac{\gamma^{\prime \prime} H^{4}}{4 \rho_{2}} \\
& +2 k_{11} \zeta_{2} \frac{Q_{3}^{(2)}}{\mu_{1}} \frac{\gamma^{\prime \prime \prime} H^{4}}{4 P_{2}} \\
& -2 k_{11} \frac{\gamma H^{4}}{4 Q_{3}^{(2)}} \frac{Q_{3}^{(2)}}{\mu_{1}}, \quad \zeta_{2}=\frac{P_{2}}{2 Q_{3}^{(2)}},
\end{aligned}
$$

$$
\begin{aligned}
& Y_{5}=\frac{Q_{1}^{(2)}}{\mu_{1} \mathrm{e}^{a H}} \\
& \frac{\left(\mu_{21}\left(\alpha_{11} \beta_{11}+\beta_{12} \alpha_{12}\right)+\mu_{22}\left(\alpha_{11} \beta_{12}-\alpha_{12} \beta_{11}\right)\right)}{\left(\mu_{21}^{2}+\mu_{22}^{2}\right)\left(\alpha_{11}^{2}+\alpha_{12}^{2}\right)}, \\
& Y_{6}=\frac{Q_{1}^{(2)}}{\mu_{1} \mathrm{e}^{a H}} \\
& \frac{\left(\mu_{21}\left(\alpha_{11} \beta_{12}-\beta_{11} \alpha_{12}\right)-\mu_{22}\left(\alpha_{11} \beta_{11}+\alpha_{12} \beta_{12}\right)\right)}{\left(\mu_{21}^{2}+\mu_{22}^{2}\right)\left(\alpha_{11}^{2}+\alpha_{12}^{2}\right)}, \\
& \alpha_{15}=\frac{\mu_{21}\left(\beta_{13} \alpha_{13}-\alpha_{14} \beta_{14}\right)-\mu_{22}\left(\beta_{14} \alpha_{13}+\alpha_{14} \beta_{13}\right)}{\left(\beta_{13}^{2}+\beta_{14}^{2}\right)\left(\alpha_{13}^{2}+\alpha_{14}^{2}\right)\left(\mu_{21}^{2}+\mu_{22}^{2}\right)}, \\
& \alpha_{16}=\frac{\mu_{21}\left(\beta_{14} \alpha_{13}+\alpha_{13} \beta_{14}\right)+\mu_{22}\left(\beta_{13} \alpha_{13}-\alpha_{14} \beta_{14}\right)}{\left(\beta_{13}^{2}+\beta_{14}^{2}\right)\left(\alpha_{13}^{2}+\alpha_{14}^{2}\right)\left(\mu_{21}^{2}+\mu_{22}^{2}\right)}, \\
& \alpha_{13}=\alpha_{11}\left(\alpha_{11}\left(\alpha_{11}^{2}-\alpha_{12}^{2}\right)-2 \alpha_{11} \alpha_{12}^{2}\right) \\
& -\alpha_{12}\left(\alpha_{12}\left(\alpha_{11}^{2}-\alpha_{12}^{2}\right)+2 \alpha_{11} \alpha_{12}^{2}\right) \text {, } \\
& \beta_{13}=\beta_{11}^{2}-\beta_{12}^{2} \text {, } \\
& \alpha_{14}=\alpha_{12}\left(\alpha_{11}\left(\alpha_{11}^{2}-\alpha_{12}^{2}\right)-2 \alpha_{11} \alpha_{12}^{2}\right) \\
& +\alpha_{11}\left(\alpha_{12}\left(\alpha_{11}^{2}-\alpha_{12}^{2}\right)+2 \alpha_{11} \alpha_{12}^{2}\right),
\end{aligned}
$$

$\beta_{14}=2 \beta_{11} \beta_{12}$,

$\mu_{21}=\left(1-k_{11} \delta_{1}\right), \quad \mu_{22}=\delta_{1}, \quad \delta_{1}=\frac{\omega_{1} \mu_{1}^{\prime}}{\mu_{1}}$,

$\beta_{11}=\sqrt{\frac{\sqrt{\beta_{01}^{2}+\beta_{02}^{2}}+\beta_{01}}{2}}$,

$$
\beta_{12}=\sqrt{\frac{\sqrt{\beta_{01}^{2}+\beta_{02}^{2}}-\beta_{01}}{2}},
$$$$
\alpha_{11}=\sqrt{\frac{\sqrt{\alpha_{01}^{2}+\alpha_{02}^{2}}+\alpha_{01}}{2}},
$$$$
\alpha_{01}=l_{20}\left(1-k_{11}^{2}\right)-2 k_{11} l_{21}+\left(\frac{a}{2 k_{01}}\right)^{2} \text {, }
$$$$
\alpha_{02}=2 k_{11} l_{20}+l_{21}\left(1-k_{11}^{2}\right),
$$$$
l_{20}=1-l_{14}-l_{16}, l_{21}=l_{15}+l_{17} \text {, }
$$$$
\alpha_{12}=\sqrt{\frac{\sqrt{\alpha_{01}^{2}+\alpha_{02}^{2}}-\alpha_{01}}{2}},
$$$$
l_{14}=\zeta_{1} \frac{\mu_{21}}{\left(\mu_{21}^{2}+\mu_{22}^{2}\right)}, \quad l_{15}=\zeta_{1} \frac{\mu_{22}}{\left(\mu_{21}^{2}+\mu_{22}^{2}\right)} \text {, }
$$$$
\zeta_{1}=\frac{P_{1}}{2 \mu_{1}} \text {, }
$$$$
l_{16}=\frac{c^{2}}{\beta_{1}^{2}} \frac{\mu_{21}}{\left(\mu_{21}^{2}+\mu_{22}^{2}\right)}, \quad l_{17}=\frac{c^{2}}{\beta_{1}^{2}} \frac{\mu_{22}}{\left(\mu_{21}^{2}+\mu_{22}^{2}\right)},
$$$$
\beta_{01}=\frac{Q_{3}^{(2)}}{Q_{1}^{(2)}}\left(\left(1-k_{11}^{2}\right)\left(1-\zeta_{2}\right)-\frac{c^{2}}{\beta_{2}^{2}}\right) \text {, }
$$ 


$$
\begin{aligned}
& \beta_{02}=\frac{Q_{3}^{(2)}}{Q_{1}^{(2)}} 2 k_{11}\left(1-\zeta_{2}-\frac{c^{2}}{\beta_{2}^{2}}\right), \\
& k_{11}=\frac{k_{02}}{k_{01}}, \quad \omega_{1}=k_{01} c .
\end{aligned}
$$

\section{Appendix A2}

$$
\begin{aligned}
& Y_{2}^{\prime}=\left(\alpha_{15}^{\prime} X_{2}^{\prime}+\alpha_{16}^{\prime} X_{3}^{\prime}\right) X_{4}^{\prime}, \\
& Y_{3}^{\prime}=\left(\alpha_{15}^{\prime} X_{3}^{\prime}-\alpha_{16}^{\prime} X_{2}^{\prime}\right) X_{4}^{\prime}, \quad X_{4}^{\prime}=\frac{1}{(k H)^{4} \mathrm{e}^{a H}}, \\
& X_{2}^{\prime}=3 \frac{\gamma^{\prime} H^{4}}{4 Q_{1}^{(2)}} \frac{Q_{1}^{(2)}}{\mu_{1}}\left(\alpha_{11}^{\prime 2}-\alpha_{12}^{\prime 2}\right)+\frac{c^{2}}{\beta_{2}^{2}} \frac{Q_{3}^{(2)}}{\mu_{1}} \frac{\gamma^{\prime \prime} H^{4}}{4 \rho_{2}} \\
& +\zeta_{2} \frac{Q_{3}^{(2)}}{\mu_{1}} \frac{\gamma^{\prime \prime \prime} H^{4}}{4 P_{2}}-\frac{\gamma H^{4}}{4 Q_{3}^{(2)}} \frac{Q_{3}^{(2)}}{\mu_{1}}, \\
& X_{3}^{\prime}=3 \frac{\gamma^{\prime} H^{4}}{4 Q_{1}^{(2)}} \frac{Q_{1}^{(2)}}{\mu_{1}}\left(2 \alpha_{11}^{\prime} \alpha_{12}^{\prime}\right) \text {, } \\
& Y_{5}^{\prime}=\frac{Q_{1}^{(2)}}{\mu_{1} \mathrm{e}^{a H}} \\
& \frac{\left(\mu_{21}^{\prime}\left(\alpha_{11}^{\prime} \beta_{11}^{\prime}+\beta_{12}^{\prime} \alpha_{12}^{\prime}\right)+\mu_{22}^{\prime}\left(\alpha_{11}^{\prime} \beta_{12}^{\prime}-\alpha_{12}^{\prime} \beta_{11}^{\prime}\right)\right)}{\left(\mu_{21}^{\prime 2}+\mu_{22}^{\prime 2}\right)\left(\alpha_{11}^{\prime 2}+\alpha_{12}^{\prime 2}\right)}, \\
& Y_{6}^{\prime}=\frac{Q_{1}^{(2)}}{\mu_{1} \mathrm{e}^{a H}} \\
& \frac{\left(\mu_{21}^{\prime}\left(\alpha_{11}^{\prime} \beta_{12}^{\prime}-\beta_{11}^{\prime} \alpha_{12}^{\prime}\right)-\mu_{22}^{\prime}\left(\alpha_{11}^{\prime} \beta_{11}^{\prime}+\alpha_{12}^{\prime} \beta_{12}^{\prime}\right)\right)}{\left(\mu_{21}^{\prime 2}+\mu_{21}^{\prime 2}\right)\left(\alpha_{11}^{\prime 2}+\alpha_{12}^{\prime 2}\right)}, \\
& \alpha_{15}^{\prime}=\frac{\mu_{21}^{\prime}\left(\beta_{13}^{\prime} \alpha_{13}^{\prime}-\alpha_{14}^{\prime} \beta_{14}^{\prime}\right)-\mu_{22}^{\prime}\left(\beta_{14}^{\prime} \alpha_{13}^{\prime}+\alpha_{14}^{\prime} \beta_{13}^{\prime}\right)}{\left(\beta_{13}^{\prime 2}+\beta_{14}^{\prime 2}\right)\left(\alpha_{13}^{\prime 2}+\alpha_{14}^{\prime 2}\right)\left(\mu_{21}^{\prime 2}+\mu_{22}^{\prime 2}\right)} \text {, } \\
& \alpha_{16}^{\prime}=\frac{\mu_{21}^{\prime}\left(\beta_{14}^{\prime} \alpha_{13}^{\prime}+\alpha_{13}^{\prime} \beta_{14}^{\prime}\right)+\mu_{22}^{\prime}\left(\beta_{13}^{\prime} \alpha_{13}^{\prime}-\alpha_{14}^{\prime} \beta_{14}^{\prime}\right)}{\left(\beta_{13}^{\prime 2}+\beta_{14}^{\prime 2}\right)\left(\alpha_{13}^{\prime 2}+\alpha_{14}^{\prime 2}\right)\left(\mu_{21}^{\prime 2}+\mu_{22}^{\prime 2}\right)} \text {, } \\
& \alpha_{13}^{\prime}=\alpha_{11}^{\prime}\left(\alpha_{11}^{\prime}\left(\alpha_{11}^{\prime 2}-\alpha_{12}^{\prime 2}\right)-2 \alpha_{11}^{\prime} \alpha_{12}^{\prime 2}\right) \\
& -\alpha_{12}^{\prime}\left(\alpha_{12}^{\prime}\left(\alpha_{11}^{\prime 2}-\alpha_{12}^{\prime 2}\right)+2 \alpha_{11}^{\prime} \alpha_{12}^{\prime 2}\right) \text {, } \\
& \beta_{13}^{\prime}=\beta_{11}^{\prime 2}-\beta_{12}^{\prime 2}, \\
& \alpha_{14}^{\prime}=\alpha_{12}^{\prime}\left(\alpha_{11}^{\prime}\left(\alpha_{11}^{\prime 2}-\alpha_{12}^{\prime 2}\right)-2 \alpha_{11}^{\prime} \alpha_{12}^{\prime 2}\right) \\
& +\alpha_{11}^{\prime}\left(\alpha_{12}^{\prime}\left(\alpha_{11}^{\prime 2}-\alpha_{12}^{\prime 2}\right)+2 \alpha_{11}^{\prime} \alpha_{12}^{\prime 2}\right), \\
& \beta_{14}^{\prime}=2 \beta_{11}^{\prime} \beta_{12}^{\prime} \text {, } \\
& \mu_{21}^{\prime}=1, \quad \mu_{22}^{\prime}=\delta_{1}^{\prime}, \quad \delta_{1}^{\prime}=\frac{\omega \mu_{1}^{\prime}}{\mu_{1}}, \\
& \beta_{11}^{\prime}=\sqrt{\frac{\sqrt{\beta_{01}^{\prime 2}+\beta_{02}^{\prime 2}}+\beta_{01}^{\prime}}{2}}, \\
& \beta_{12}^{\prime}=\sqrt{\frac{\sqrt{\beta_{01}^{\prime 2}+\beta_{02}^{\prime 2}}-\beta_{01}^{\prime}}{2}}, \\
& \alpha_{11}^{\prime}=\sqrt{\frac{\sqrt{\alpha_{01}^{\prime 2}+\alpha_{02}^{\prime 2}}+\alpha_{01}^{\prime}}{2}},
\end{aligned}
$$

$$
\begin{aligned}
& \alpha_{12}^{\prime}=\sqrt{\frac{\sqrt{\alpha_{01}^{\prime 2}+\alpha_{02}^{\prime 2}}-\alpha_{01}^{\prime}}{2}}, \\
& \alpha_{01}^{\prime}=l_{20}^{\prime}+\left(\frac{a}{2 k}\right)^{2}, \alpha_{02}^{\prime}=l_{21}^{\prime}, \\
& l_{20}^{\prime}=1-l_{14}^{\prime}-l_{16}^{\prime}, l_{21}^{\prime}=l_{15}^{\prime}+l_{17}^{\prime}, \\
& l_{14}^{\prime}=\zeta_{1} \frac{\mu_{21}^{\prime}}{\left(\mu_{21}^{\prime 2}+\mu_{22}^{\prime 2}\right)}, \quad l_{15}^{\prime}=\zeta_{1} \frac{\mu_{22}^{\prime}}{\left(\mu_{21}^{\prime 2}+\mu_{22}^{\prime 2}\right)}, \\
& \zeta_{1}=\frac{P_{1}}{2 \mu_{1}}, \quad l_{16}^{\prime}=\frac{c^{2}}{\beta_{1}^{2}} \frac{\mu_{21}^{\prime}}{\left(\mu_{21}^{\prime 2}+\mu_{22}^{\prime 2}\right)}, \\
& l_{17}^{\prime}=\frac{c^{2}}{\beta_{1}^{2}} \frac{\mu_{22}^{\prime}}{\left(\mu_{21}^{\prime 2}+\mu_{22}^{\prime 2}\right)}, \quad \\
& \beta_{01}^{\prime}=\frac{Q_{3}^{(2)}}{Q_{1}^{(2)}}\left(\left(1-\zeta_{2}\right)-\frac{c^{2}}{\beta_{2}^{2}}\right), \quad \beta_{02}^{\prime}=0, \quad \omega=k c .
\end{aligned}
$$

\section{References}

Achenbach J 2012 Wave propagation in elastic solids; Elsevier.

Biot M A 1962 Mechanics of deformation and acoustic propagation in porous media; J. Appl. Phys. 33(4) 1482-1498.

Caloi P 1948 Comportement des cndes de Rayleigh dans un milreu firmoélastique indéfini; Bur. Cent. Seismol. Inb. 17 89-108

Chaudhary S, Kaushik V P and Tomar S K 2005 Transmission of shear waves through a self-reinforced layer sandwiched between two inhomogeneous viscoelastic halfspaces; Int. J. Mech. Sci. 47(9) 1455-1472.

Carcione J M 1990 Wave propagation in anisotropic linear viscoelastic media: Theory and simulated wavefields; Geophys. J. Int. 101(3) 739-750.

Chatterjee M and Chattopadhyay A 2015 Propagation, reflection, and transmission of SH-waves in slightly compressible, finitely deformed elastic media; J. Appl. Math. Mech. 36(8) 1045-1056.

Chattopadhyay A, Gupta S, Sharma V K and Kumari P 2010 Propagation of shear waves in viscoelastic medium at irregular boundaries; Acta Geophys. 58(2) 195-214.

Chattopadhyay A, Gupta S, Singh A K and Sahu S A 2011 Effect of point source, self-reinforcement and heterogeneity on the propagation of magnetoelastic shear wave; $A p p l$. Math. 2(03) 271.

Daley P F and Krebes E S 2004 SH wave propagation in viscoelastic media; Stud. Geophys. Geod 48(3) 563-587.

Gubbins D 1990 Seismology and plate tectonics; Cambridge University Press.

Kakar R and Kakar S 2016 Love wave in a Voigt-type viscoelastic heterogeneous layer overlying heterogeneous viscoelastic half-space; Int. J. Geomech. 17(1) 06016009.

Kaur T, Sharma S K and Singh A K 2017 Shear wave propagation in vertically heterogeneous viscoelastic layer over a micropolar elastic half-space; Mech. Adv. Mater. Struc. 24(2) 149-156.

Kundu S, Gupta S, Vaishnav P K and Manna S 2016 Propagation of Love waves in a heterogeneous medium over an inhomogeneous half-space under the effect of point source; J. Vib. Control. 22(5) 1380-1391. 
Kundu S, Manna S and Gupta S 2015 Propagation of $\mathrm{SH}$-wave in an initially stressed orthotropic medium sandwiched by a homogeneous and an inhomogeneous semi-infinite media; Math. Methods Appl. Sci. 38(9) 1926-1936.

Romeo M 2003 Interfacial viscoelastic SH waves; Int. J. Solids Struct. 40(9) 2057-2068.

Sahu S A, Saroj P K and Dewangan N 2014 SH-waves in viscoelastic heterogeneous layer over half-space with selfweight; Arch. Appl. Mech. 84(2) 235-245.
Sharma M D 2004 Strains of scattering of near-field of a point source; J. Earth Syst. Sci. 113(2) 247-257.

Vavryčuk V 2008 Velocity attenuation, and quality factor in anisotropic viscoelastic media: A perturbation approach velocity, attenuation, and quality factor; Geophysics 73(5) D63-D73.

Vlaar N J 1966 The field from an SH point source in a continuously layered inhomogeneous half-space: II. The field in a half-space; Bull. Seismol. Soc. Am. 56(6) 1305-1315.

Corresponding editor: Arkoprovo BiswaS 